\title{
T-Matrix Approach to Quarkonium Correlation Functions in the QGP
}

\author{
D. Cabrera and R. Rapp \\ Cyclotron Institute and Physics Department, \\ Texas AEMM University, College Station, Texas 77843-3366, U.S.A.
}

(Dated: October 30, 2018)

\begin{abstract}
We study the evolution of heavy quarkonium states with temperature in a Quark Gluon Plasma (QGP) by evaluating the in-medium $Q-\bar{Q} T$-matrix within a reduced Bethe-Salpeter equation in both $S-$ and $P$-wave channels. The underlying interaction kernel is extracted from recent finite-temperature QCD lattice calculations of the singlet free energy of a $Q-\bar{Q}$ pair. The bound states are found to gradually move above the $Q-\bar{Q}$ threshold after which they rapidly dissolve in the hot system. The $T$-matrix approach is particularly suited to investigate these mechanisms as it provides a unified treatment of bound and scattering states including threshold effects and the transition to the (perturbative) continuum. We apply the $T$-matrix to calculate $Q-\bar{Q}$ spectral functions as well as pertinent Euclidean-time correlation functions which are compared to results from lattice QCD. A detailed analysis reveals large sensitivities to the interplay of bound and scattering states, to temperature dependent threshold energies and to the "reconstructed" correlator used for normalization. We furthermore investigate the impact of finite-width effects on the single-quark propagators in the QGP as estimated from recent applications of heavy-quark rescattering to RHIC data.
\end{abstract}

PACS numbers: 25.75.Dw, 12.38.Gc, 24.85.+p, 25.75.Nq 


\section{INTRODUCTION}

Bound states of heavy quarks (charm and bottom, $Q=c, b$ ) have long been recognized as valuable objects for spectroscopy in Quantum Chromodynamics (QCD), thereby illuminating the nature of the static quark-antiquark potential (cf. Ref. [1] for a recent comprehensive overview). This opportunity carries over when embedding quarkonia into hot and/or dense matter, providing a rich laboratory for the study of medium modifications. The latter include (Debye-) color-screening of the $Q-\bar{Q}$ interaction, dissociation reactions induced by constituents of the medium, and the change in thresholds caused by mass (or width) modifications of open heavy-flavor states ( $D$ and $B$ mesons or $c$ and $b$ quarks). The challenge is to develop a theoretical framework that allows a comprehensive description of heavy quarkonia in the Quark-Gluon Plasma and their production in ultrarelativistic heavy-ion collisions.

Lattice QCD (1QCD) calculations have made substantial progress in characterizing in-medium quarkonium properties from first principles. In particular, it has been found that ground state charmonia [2, 3, 4] and bottomonia [5] do not dissolve until significantly above the critical temperature, $T_{c}$. This finding has been qualitatively supported in model calculations based on potentials extracted from lQCD, using either a Schrödinger equation to solve the bound state problem [6, 7, , $8,[9]$, or a $T$-matrix approach which simultaneously accounts for scattering states [10]. The survival of low-lying quarkonia above $T_{c}$, in connection with effects of color-screening, parton-induced dissociation and medium modified open-charm and -bottom thresholds, has recently been implemented for heavy-ion collisions [11, 12].

A more quantitative (and reliable) comparison of model calculations to lQCD can be performed at the level of (spacelike) Euclidean-time correlation functions [13, 14]. The latter are directly evaluated in LQCD with good accuracy, while the conversion of (timelike) spectral functions as evaluated in model approaches merely involves a straightforward convolution with a thermal weight function (as opposed to an inverse integral transform when going from Euclidean to Minkowski space). One of the challenges in such studies is that the model calculations need to describe not only the bound-state part of the spectral function but also its continuum part as well as threshold effects. In Ref. [9] a quantitative calculation of Euclidean correlators was performed using temperature-dependent heavy-quark potentials in a Schrödinger equation. The latter has been used to determine the bound-state spectrum in $\delta$-function approximation (characterized by a binding energy and amplitude (or decay constant)), while the (onset of the) continuum was approximated with perturbation theory. While general trends of the lQCD correlators were captured, significant discrepancies were established especially in the $S$-wave charmonium channels $\left(\eta_{c}\right.$ and $\left.J / \psi\right)$. In particular, the importance of a reliable treatment of the continuum threshold was recognized.

In the present paper we evaluate charmonium and bottomonium correlators using a different method. The basic input are still in-medium $Q-\bar{Q}$ potentials as estimated from 1QCD, but we will employ these within a scattering equation to calculate the in-medium $Q-\bar{Q} T$-matrix [10]. The main advantage of the $T$-matrix approach is that it simultaneously incorporates bound and scattering states based on the same interaction. Especially for situations of dissolving bound states (as expected for the problem at hand) the $T$-matrix provides a more comprehensive, and thus more reliable, description of the underlying nonperturbative effects. At the correlator level, the high-energy limit can be recovered by appropriate normalization of the uncorrelated (perturbative) limit, and no decomposition into bound-state and continuum parts is necessary. In addition, the $T$-matrix equation allows for a straightforward implementation of in-medium single-particle (quark) properties via pertinent self-energy insertions in the two-particle Green's function, which we will also investigate.

Our article is organized as follows: in Sec. III we recall the basic set-up of, and input to, the two-body scattering equation, including partial-wave expanded potentials and single-quark 
selfenergy insertions. In Sec. III we evaluate the finite-temperature $T$-matrices for $S$ - and $P$-wave quarkonia; we first extract heavy-quark potentials from lQCD in Sec. IIIA including a discussion of its short- and large-distance limits and relations to single-quark properties, followed by our baseline results for the finite-temperature quarkonium $T$-matrices in Sec. IIIB. In Sec. IV] the latter are first employed to construct pertinent spectral functions (Sec. IVA), followed by a calculation of Euclidean correlators (Sec. IVB) and a discussion of their properties in comparison to other model and lQCD results (Sec. IVC). Sec. $\mathrm{V}$ contains our conclusions and an outlook.

\section{SCATTERING EQUATION AND IDENTIFICATION OF BOUND STATES}

We here summarize the main features of the $T$-matrix approach to study quark-antiquark interactions in the QGP, as employed in Ref. [10]. It utilizes a three-dimensional reduction of the Bethe-Salpeter equation which neglects virtual particle-antiparticle loops and amounts to resumming the scattering series in ladder approximation. The pertinent Lippmann-Schwinger equation for the off-shell $T$-matrix in a given partial-wave channel (specified by angular momentum $l$ ) reads $^{1}$

$$
T_{l}\left(E ; q^{\prime}, q\right)=V_{l}\left(q^{\prime}, q\right)+\frac{2}{\pi} \int_{0}^{\infty} d k k^{2} V_{l}\left(q^{\prime}, k\right) G_{\bar{Q} Q}(E ; k) T_{l}(E ; k, q)\left[1-2 f^{Q}\left(\omega_{k}\right)\right],
$$

where $q\left(q^{\prime}\right)$ are the incoming (outgoing) relative quark three-momenta in the center of mass (CM) frame and $E$ is the $\mathrm{CM}$ energy ${ }^{2}$. Eq. (II) is written for vanishing total 3-momentum of the heavy-quark pair, which gives the above (simple) form of the Pauli blocking factor with $f^{Q}(\omega)=$ $[\exp (\omega / T)+1]^{-1}$. The intermediate two-particle propagator is evaluated in the BlankenbeclerSugar reduction scheme [15] (uncertainties due to other reduction schemes have been checked to be small [10]),

$$
G_{\bar{Q} Q}(E ; k)=\frac{m^{2}}{\omega_{k}} \frac{1}{s / 4-\omega_{k}^{2}-2 i \omega_{k} \operatorname{Im} \Sigma\left(\omega_{k}, k\right)},
$$

where $\omega_{k}$ is the solution of the quark dispersion relation,

$$
\omega_{k}=\sqrt{m^{2}+k^{2}}+\operatorname{Re} \Sigma\left(\omega_{k}, k\right)
$$

with a quark-mass term $(m)$ and selfenergy $(\Sigma)$ to be discussed below. The interaction kernel of the scattering equation, $V_{l}\left(q^{\prime}, q\right)$, is provided by the heavy-quark potential in momentum space. It follows from a Fourier transformation of the coordinate-space potential, $V(r)$, which we obtain from lQCD calculations as elaborated in Sec. IIIA below. The components of the potential in the partial-wave basis are given by

$$
V_{l}\left(q^{\prime}, q\right)=\frac{1}{8 \pi} \int_{-1}^{+1} d u_{q^{\prime} q} V\left(\vec{q}^{\prime}, \vec{q}\right) P_{l}\left(u_{q^{\prime} q}\right)=\frac{1}{8 \pi} \int_{-1}^{+1} d u_{q^{\prime} q} P_{l}\left(u_{q^{\prime} q}\right) \int d^{3} r V(r) e^{i\left(\vec{q}-\vec{q}^{\prime}\right) \vec{r}},
$$

with $P_{l}(x)$ the Legendre polynomial of degree $l$ and $u_{q^{\prime} q}=\cos \left\langle\widehat{\vec{q}, \vec{q}^{\prime}}\right\rangle$.

The $T$-matrix equation (11) is solved with the algorithm of Haftel and Tabakin [16]: after discretizing the momentum integration, Eq. (11) is converted into a matrix equation,

$$
\sum_{k=1}^{N} \mathcal{F}(E)_{i k} T(E)_{k j}=V_{i j},
$$

\footnotetext{
${ }^{1}$ The partial wave expansion reads $T=4 \pi \sum_{l}(2 l+1) T_{l} P_{l}(\cos \theta)$, and similarly for the potential.

2 The on-shell $T$-matrix is defined for $q=q^{\prime}$ and $E=\sqrt{s}=2 \omega_{q}$, with $\omega_{q}$ the (relativistic) on-shell heavy-quark energy.
} 
where, schematically, $\mathcal{F}=1-w V G_{\bar{Q} Q}\left[1-2 f^{Q}\right]$ (with $w$ denoting an integration weight). The solution for the $T$-matrix then follows from matrix inversion.

To assess the presence of heavy quark-antiquark bound states, the $T$-matrix has to be studied below the $Q-\bar{Q}$ threshold, $E_{t h}=2 \omega_{q=0}$. The non-relativistic potential, $V_{l}\left(q^{\prime}, q\right)$, is only defined for real external three-momenta, and therefore an evaluation of $T$ below the $Q-\bar{Q}$ threshold requires a prescription for the subthreshold continuation of the potential. For $S$-wave scattering we follow the standard convention of setting the momenta to zero [17],

$$
T_{0}\left(E<E_{t h}\right)=T_{0}\left(E ; q^{\prime}=q=0\right) .
$$

The reliability of this continuation can be checked by exploiting the (numerical) matrix form of the scattering equation. Since a bound state corresponds to a pole of the amplitude on the real energy axis below threshold, it follows that the determinant of the transition matrix $\mathcal{F}$ must vanish at the bound-state energy [16],

$$
\operatorname{det} \mathcal{F}(E)=0, E<E_{t h} \text {. }
$$

A similar condition arises from the solution of the Schrödinger equation for the bound state problem [16, 18]. This is equivalent to finding the zeroes of the Jost function in scattering ( $S$-matrix) theory. For $P$-wave states, the potential is proportional to the in- and outgoing quark momentum and therefore the continuation in Eq. (6) cannot be applied. However, the condition Eq. (7) remains valid and will be used to determine $P$-wave bound states.

The quark selfenergy figuring into the two-particle propagator, Eq. (2), receives contributions from interactions with (light) anti-/quarks and gluons in the heat bath. In Ref. [10] this was schematically written as

$$
\Sigma=\widetilde{\Sigma}+\int f^{q} T_{Q q} S_{q}
$$

where $\widetilde{\Sigma}$ denotes the gluonic piece and the second term involves the heavy-light quark $T$-matrix closed by a light-quark propagator, $S_{q}$, and a thermal distribution, $f^{q}$. Rather than using an explicit model calculation for $T_{Q q}$ [19], in the present work we will constrain ourselves to the following levels of approximation: (a) a fixed heavy-quark mass $m$ (i.e., $\operatorname{Re} \Sigma=0$ ) together with a small imaginary part, $\operatorname{Im} \Sigma=-0.01 \mathrm{GeV}$, mostly for numerical purposes (to avoid $\delta$-function like bound states in the $T$-matrix); (b) a temperature dependent heavy-quark mass as estimated from the asymptotic value of the IQCD heavy-quark internal energies; (c) a heavy-quark width as calculated in an effective model for resonance (plus perturbative gluon) interactions in the QGP [20], which has been shown to give reasonable agreement with data on suppression and elliptic flow of semileptonic electron spectra from heavy-quark decays in Au-Au collisions at RHIC [21]. We also note that interactions with heavy anti-/quarks from the medium can be safely neglected due to the smallness of the number of $Q$ 's in the system. This is different to (and simpler than) the situation of the light-quark selfenergy which, in turn, figures into the calculation of the $T$-matrix, constituting a self-consistency problem as has been evaluated, e.g., in Ref. [10].

\section{TEMPERATURE EVOLUTION OF HEAVY QUARKONIUM T-MATRICES}

\section{A. Quark-Antiquark Potential from Lattice QCD}

For the driving kernel of the scattering equation we focus on heavy-quark potentials from lQCD. There is an ongoing discussion as to how to properly extract them from the (static) heavy-quark 
free energy. Using directly the latter leads to a dissociation temperature of ground-state charmonia of about $T_{\text {diss }} \simeq 1.1 T_{c}$ [22], while the lattice analysis of spectral functions suggests that $\eta_{c}$ and $J / \psi$ survive up to around $2 T_{c}$. These higher values for $T_{\text {diss }}$ can be recovered if the (color-singlet) internal energy,

$$
U_{1}=F_{1}-T \frac{d F_{1}}{d T}
$$

is identified with the $Q-\bar{Q}$ potential $[7,8,19,10]$. Another possibility, namely a suitable linear combination of $U_{1}$ and $F_{1}$, has been suggested in Ref. [7]. The temperature derivative of discrete lQCD "data points" involved in the extraction of $U_{1}$ induces significant uncertainty which is comparable to, e.g., the difference between quenched and unquenched results (after rescaling of the critical temperature), as studied in Ref. [10]. In view of this situation, one has to accept a certain level of uncertainty in the potential. To adequately account for this, we adopt two versions of the internal energy, Eq. (9), as the potential: (i) based on fits to the two-flavor lQCD results for the free energy from Refs. [23, 24], we explicitely perform the temperature derivative in Eq. (9); (ii) we directly fit the two-flavor 1QCD internal energy data as extracted in the calculation of Ref. [25]. Further investigations of the impact of using different definitions of the $Q-\bar{Q}$ potential will be considered in future work.

The long-distance limit of the potential entering Eq. (11) has to be normalized to zero to ensure the convergence of the scattering equation,

$$
V(r, T)=U_{1}(r, T)-U_{1}^{\infty}(T),
$$

with $U_{1}^{\infty}(T) \equiv U_{1}(r \rightarrow \infty, T)$. In Refs. [7, 8, 9, 26, 27] the linearity of the Schrödinger equation is exploited to trade the internal energy at infinite distance into the energy of the bound states. In Ref. [9], $U_{1}^{\infty}(T)$ is interpreted as an effective in-medium contribution to the quark mass, $\Delta m_{Q}(T)=$ $U_{1}^{\infty}(T) / 2$ and implemented as a change in the $Q-\bar{Q}$ threshold energy, $E_{t h}=2 m_{Q}+U_{1}^{\infty}(T)$, in the calculation of the mesonic spectral function. It is argued that this correction should not modify the mass operator in the Schrödinger equation since quarks inside a bound state do not "sense" the medium and therefore should not be subject to medium-induced mass modifications. This ambiguity in the interpretation of the internal energy at infinite distance can be resolved within the many-body scattering equation approach. The interaction of the quark with the surrounding medium induces a selfenergy which is encoded in an effective mass change $\left(\Delta m_{Q}=\operatorname{Re} \Sigma\right)$. As such, this medium effect has to be included in the two-particle propagator, Eq. (2), and therefore contributes in a nonlinear way as it is iterated to all orders in the scattering equation series. As we discuss in the following sections, this not only modifies the $Q-\bar{Q}$ threshold energy but the evolution of the binding energy (total mass of the bound state) with temperature, leading to different dissociation temperatures when the effective in-medium mass is considered.

Another intriguing problem regarding the interpretation of $U_{1}^{\infty}$ as an in-medium quark mass (or selfenergy) is its possible momentum dependence. Whereas at infinite separation (low momentum transfer) $U_{1}^{\infty}$ operates as $\Delta m_{Q}$, at short distances (high momentum transfer) the $Q-\bar{Q}$ system is no longer sensitive to the medium, and the quark mass should be unaffected. Implementing such a momentum dependent quark selfenergy would require a microscopic treatment of the mass subtraction of the internal energy. Instead, we will consider two limiting scenarios, namely (a) no in-medium mass correction and (b) in-medium effective mass as given by $\Delta m_{Q}(T)=U_{1}^{\infty}(T) / 2$.

Finally, for the potentials in momentum space we introduce a relativistic correction motivated by the velocity-velocity (Breit) interaction in electrodynamics [28], see also Ref. [10]. It amounts to the following factor:

$$
V\left(q^{\prime}, q\right) \rightarrow V\left(q^{\prime}, q\right)\left[1+q^{\prime 2} / \omega_{q^{\prime}}^{2}\right]^{1 / 2}\left[1+q^{2} / \omega_{q}^{2}\right]^{1 / 2}
$$


The two potentials used in this work are summarized in Fig. 1, For case (i) discussed above (extraction from the 1QCD free energy), it evolves rather smoothly with temperature (left panel), while for case (ii) (1QCD internal energy) the potential is initially more attractive but weakens rapidly with temperature (right panel) for $T \lesssim 1.5 T_{c}$ and slows down thereafter. The rapid decrease is mostly induced by $U_{1}^{\infty}(T)$, see also Ref. [25].
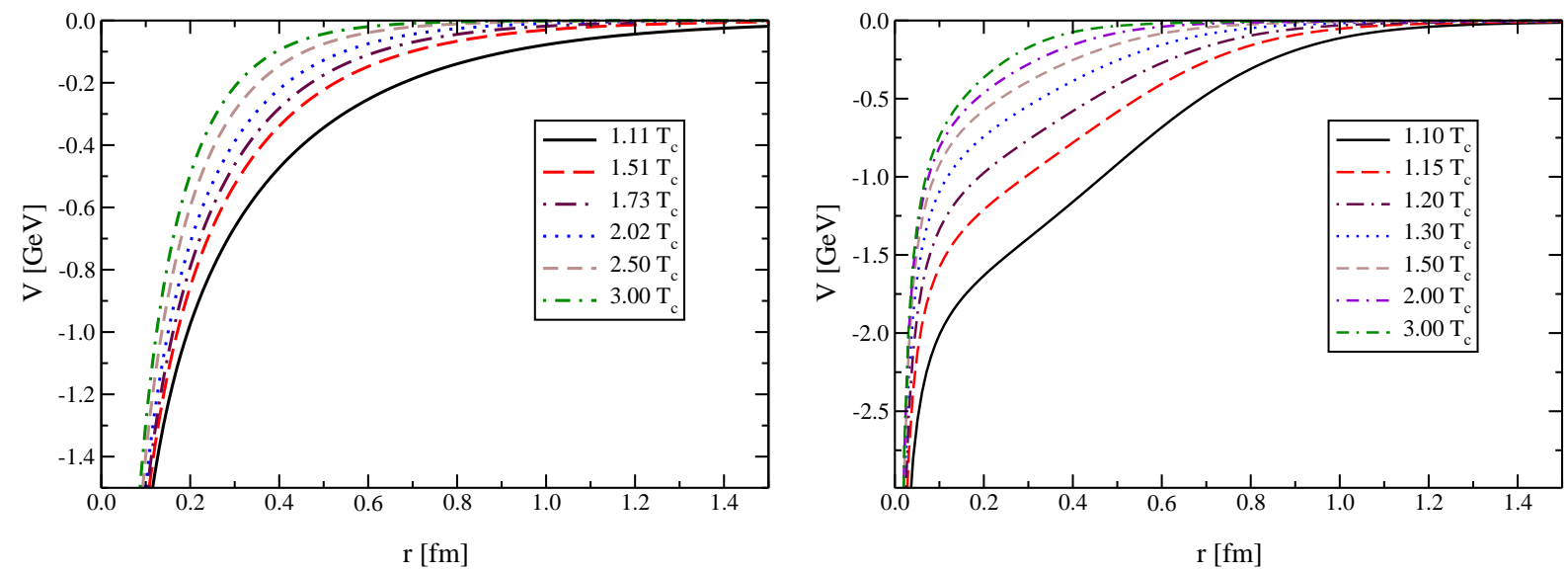

FIG. 1: $Q-\bar{Q}$ color-singlet potential for several temperatures above $T_{c}$, as defined in Eq. (10); left panel: based on $F_{1}$ from Refs. [23, 24] in connection with Eq. (9); right panel: based on $U_{1}$ as directly evaluated in $1 Q C D$ in Ref. [25].

\section{B. Quarkonium $T$-Matrices in the QGP}

We now turn to the numerical results for the finite-temperature $T$-matrices in the $c-\bar{c}$ and $b-\bar{b}$ sectors, obtained by solving the scattering equation (11) in both $S$ - and $P$-wave channels as described above.

\section{S-Wave States}

In a first step, we consider the case of narrow quark spectral functions with $\operatorname{Im} \Sigma=-10 \mathrm{MeV}$ (for numerical purposes) and constant (temperature-independent) heavy-quark masses $(\operatorname{Re} \Sigma=0)$. The latter are fixed so that the corresponding ground states are located approximately at their vacuum masses for the lowest considered temperature $\left(T=1.1 T_{c}\right)$, yielding $m_{c}=1.7 \mathrm{GeV}$ and $m_{b}=5.15 \mathrm{GeV}$.

Fig. 2 summarizes the on-shell $S$-wave $c-\bar{c}$ scattering amplitude as a function of $\mathrm{CM}$ energy, for several temperatures from $1.1 T_{c}$ to $3.3 T_{c}$, as well as the determinant function $\operatorname{det} \mathcal{F}(E)$ (in arbitrary units). Since we do not include the hyperfine (spin-spin) interactions, $\eta_{c}\left(\eta_{b}\right)$ and $J / \psi$ $(\Upsilon)$ states are degenerate. At the lowest temperature, we recover the charmonium ground state at $E \approx 3.0 \mathrm{GeV}$, and also find a cusp at the $c \bar{c}$ threshold energy indicating that the first excited state (ascribed to the $\psi^{\prime}$ ) has just melted. The determinant function, $\operatorname{det} \mathcal{F}(E)$, vanishes exactly at the ground state energy coinciding with $\operatorname{Re} T(E)=0$, thus corroborating our subthreshold 


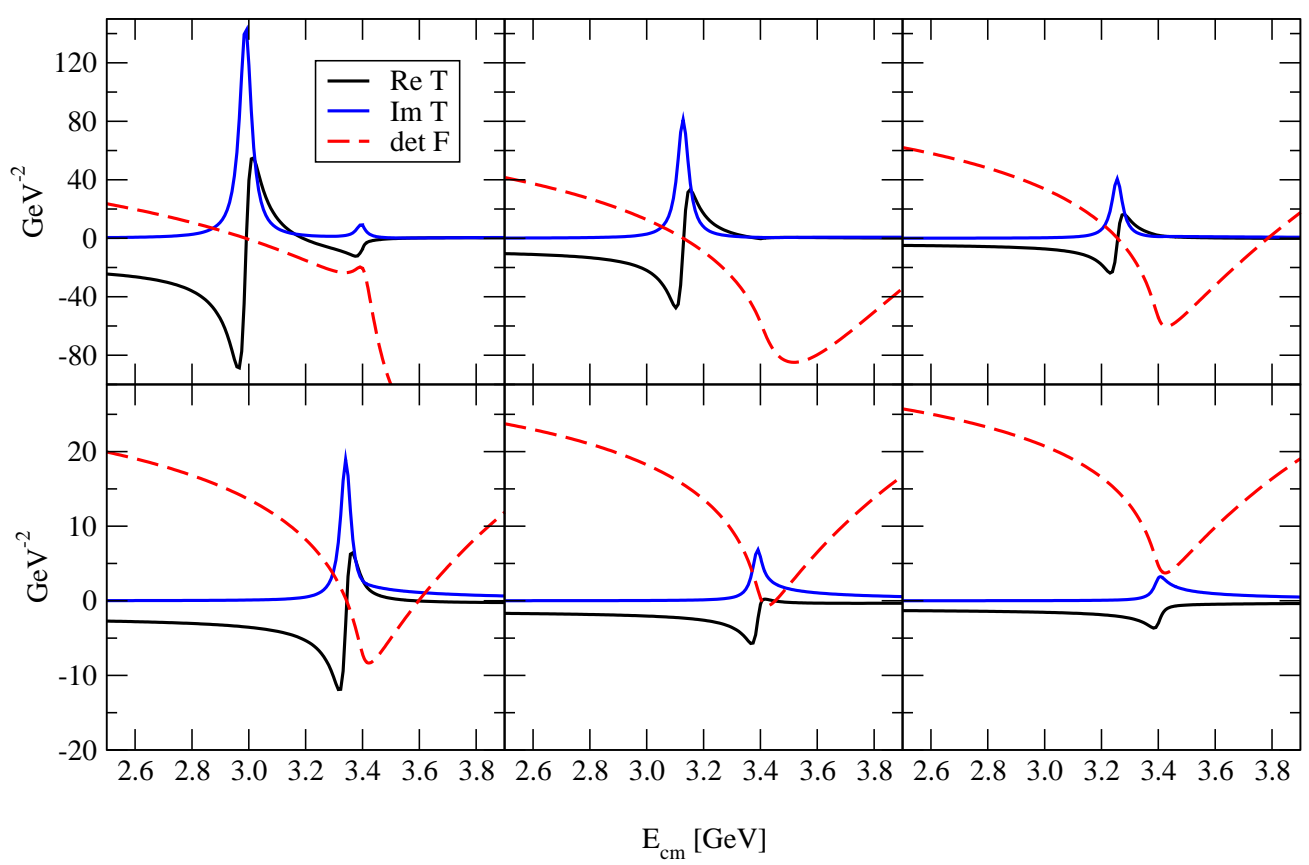

FIG. 2: Real and imaginary parts of the $T$-matrix for $S$-wave $c-\bar{c}$ scattering in the QGP based on potentials derived from the lQCD free energy of Ref. 24]. Also shown is the determinant function det $\mathcal{F}$ (dashed line, arbitrary units). From left to right and up to down the temperatures are $(1.1,1.5,2.0,2.5,3.0,3.3) T_{c}$.

continuation of the $T$-matrix, Eq. (6). For higher energies $\operatorname{det} \mathcal{F}(E)$ approaches zero again, but as soon as the threshold is reached it deviates indicating that the first excited state has already crossed into the continuum spectrum ${ }^{3}$. As the temperature is increased, the bound charmonium state gradually moves toward threshold, indicating a reduction of its binding energy. At the same time, the magnitude of the $T$-matrix is appreciably reduced. The $J / \psi(1 S)$ survives as a bound state well beyond $T_{c}$, eventually crossing the threshold at about $(2.8-3.0) T_{c}$, after which it turns into a resonance and rapidly melts in the hot system ${ }^{4}$. Our results agree reasonably well with those of Ref. [10], with some differences in size and shape of the scattering amplitude, in particular a larger dissociation temperature. This is mostly due to a different parametrization of the $Q-\bar{Q}$ potential (reflecting the uncertainties in a derivation of the $Q-\bar{Q}$ internal energy from a fit to the free energy, cf. Sec. IIIB 4 below) and a different choice of the two-particle propagator, which implies deviations at order $\mathcal{O}(\mathrm{p} / \mathrm{m})$. The robustness of this approach to dynamically generate quarkonium bound and scattering states and their evolution with temperature is confirmed and extended in the following to study other quarkonium states.

The results for $S$-wave $b-\bar{b}$ scattering are depicted in Fig. 3. At the lowest temperature the $T$-matrix exhibits two bound states, as well as the remnant of a third one. The bound-state locations are again quantitatively confirmed by the vanishing determinant of the transition matrix

\footnotetext{
${ }^{3}$ Strictly speaking, $\mathcal{F}(E)$ is not a purely real function since we have included a small imaginary part in the twoparticle propagator. More precisely, the bound-state condition, Eq. (7), reads $\operatorname{Re}\{\operatorname{det} \mathcal{F}(E)\}=0$. We are plotting $\operatorname{Re}\{\operatorname{det} \mathcal{F}(E)\}$ in Figs. 2,3 and 4 .

${ }^{4}$ We refer to a state "melting" or "dissolving" when the scattering amplitude is strongly broadened and diminished corresponding to a loss of the resonant structure.
} 


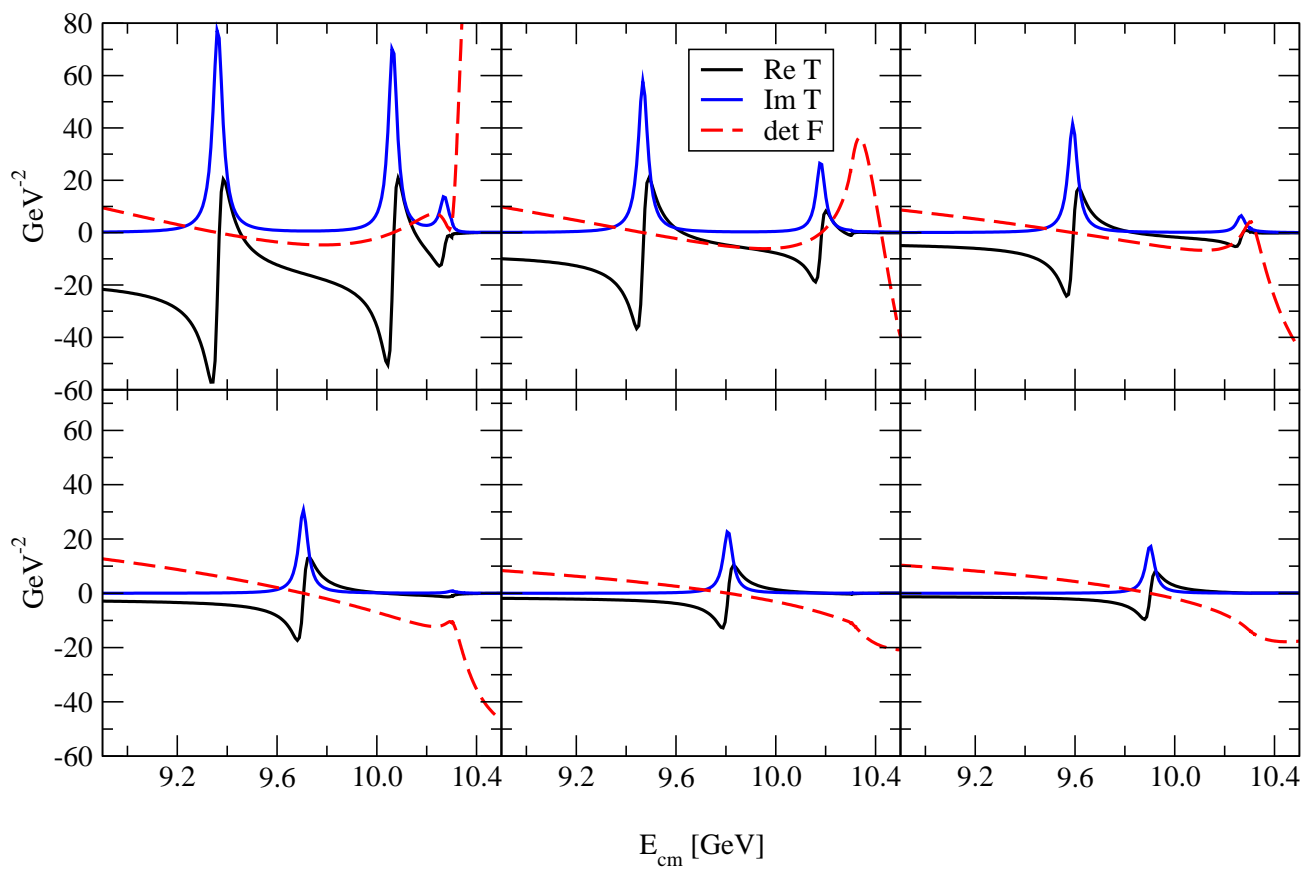

FIG. 3: Same as in Fig. 2 but for $S$-wave $b-\bar{b}$ scattering. From left to right and up to down the temperatures are $(1.1,1.5,1.8,2.1,2.7,3.5) T_{c}$.
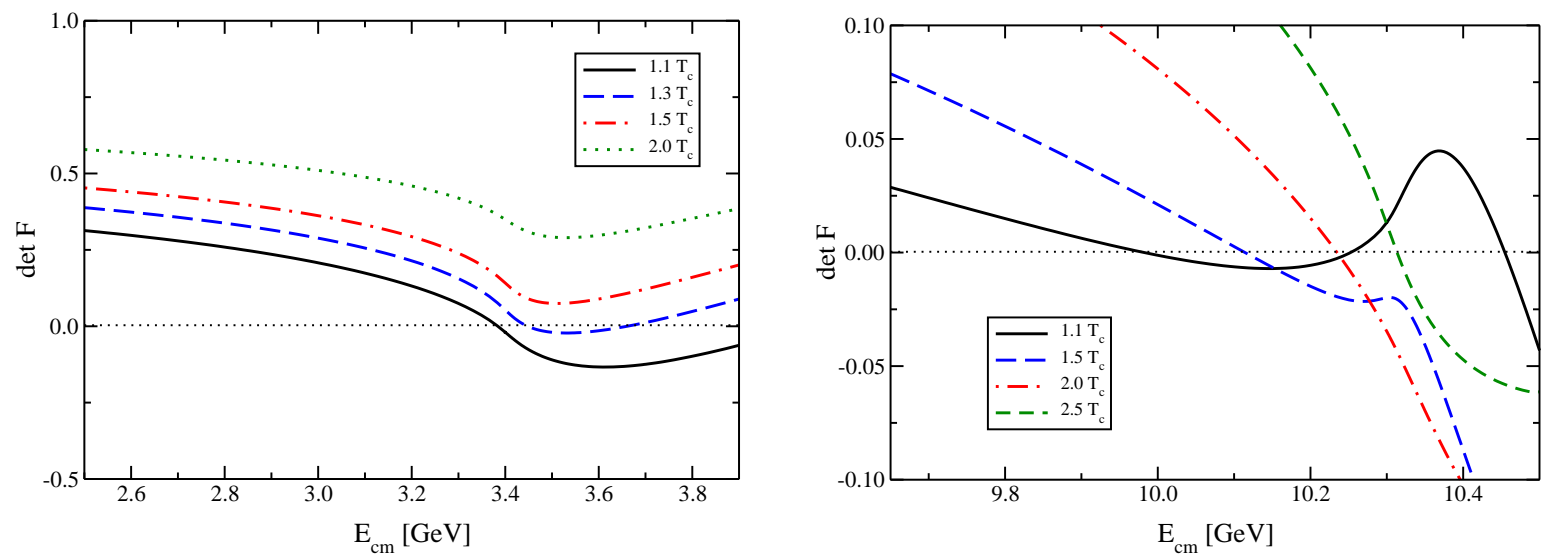

FIG. 4: Pole identification function $(\operatorname{det} \mathcal{F})$ for charmonium (left) and bottomonium (right) $P$-wave scattering at several temperatures.

(dashed lines), while it barely reaches zero at the location of the third structure in the $T$-matrix, which carries much smaller strength, indicating that it has practically melted in the medium. The two bound states at $E \approx 9.35,10.05 \mathrm{GeV}$ are ascribed to the ground and first-excited bottomonium states $\Upsilon(1 S), \eta_{b}$ and $\Upsilon(2 S), \eta_{b}^{\prime}$, respectively. The $\Upsilon(2 S)$ moves above the $b-\bar{b}$ threshold at $T \approx$ $1.8 T_{c}$, whereas the $1 S$ state survives in the QGP until much higher temperatures, beyond $T \approx$ $3.5 T_{c}$.

2. P-Wave States 


\begin{tabular}{c||ccccc}
$T / T_{c}$ & 1.1 & 1.3 & 1.5 & 2 & 2.3 \\
\hline \hline$M\left[\chi_{c}(1 P)\right]$ & 3.38 & - & - & - & - \\
\hline$E_{B}\left[\chi_{c}(1 P)\right]$ & $\approx 0$ & - & - & - & - \\
\hline \hline$M\left[\chi_{b}(1 P)\right]$ & 9.95 & 10.05 & 10.11 & 10.23 & 10.30 \\
\hline$E_{B}\left[\chi_{b}(1 P)\right]$ & 0.35 & 0.25 & 0.19 & 0.07 & $\approx 0$ \\
\hline$M\left[\chi_{b}(2 P)\right]$ & 10.25 & 10.30 & - & - & - \\
\hline$E_{B}\left[\chi_{b}(2 P)\right]$ & 0.05 & $\approx 0$ & - & - & - \\
\hline \hline
\end{tabular}

TABLE I: Summary of masses and binding energies (in $[\mathrm{GeV}]$ ) for $P$-wave quarkonia in the QGP as extracted from the finite-temperature $T$-matrix determinant, Eq. (7).

Next we study $Q-\bar{Q}$ scattering in a relative $P$-wave. In order to assess the formation of bound states in this channel we rely on the condition in Eq. (77) and determine the zeroes of $\operatorname{det} \mathcal{F}(E)$, which is plotted in Fig. 4 for several temperatures for both charm and bottom. We only find one $c-\bar{c}$ bound state at the lowest temperature $\left(T=1.1 T_{c}\right)$, at $E \approx 3.4 \mathrm{GeV}$ (just below threshold), which we associate with the $1 P$ charmonium, $\chi_{c}$. As the temperature increases, the $\chi_{c}$ state rapidly shifts into the continuum.

The $P$-wave $b-\bar{b}$ system exhibits two bound states at the lowest temperature, which we may identify with the $\chi_{b}(1 P)$ and $\chi_{b}(2 P)$ as their energies $(E=9.95,10.25 \mathrm{GeV})$ are close to the nominal values in the vacuum. The $\chi_{b}(2 P)$ state moves beyond threshold for $T \approx 1.3 T_{c}$ and the $(1 P)$ state for $T \approx 2.3 T_{c}$. Both the mass and the binding energies, $\left(E_{B}=E_{t h}-M\right)$, of the $P$-wave states are summarized in Tab. I for several temperatures.

\section{Continuum Scattering}

The $T$-matrix approach also encompasses the continuum part of the spectrum. This is not easily appreciated in Figs. 2 and 3 because of the different scales of the narrow bound state signal and the amplitude above threshold. Note that the determinant of the transition matrix, det $\mathcal{F}$, vanishes for some energies above threshold (cf. Figs. 244), possibly indicating resonant scattering in the continuum. Indeed, as can be seen in Fig. 5, the $T$-matrix for $c-\bar{c} S-$ and $P$-wave scattering exhibits substantial correlations above threshold. The imaginary part shows a distorted resonant shape, which peaks at approximately the same energy where the real part vanishes. The non-perturbative effects of the $Q-\bar{Q}$ rescattering above threshold are evident as we compare the $T$-matrix to its Born approximation, $V$ (dash-dotted line in Fig. 5). We confirm that the $T$-matrix shows the expected behavior at high energies, i.e., the real part converges to the Born approximation and the imaginary part tends to vanish. Finally, Fig. 6 displays the imaginary part of the $S$-wave scattering amplitude on a logarithmic scale over a wide energy range below and above the $Q-\bar{Q}$ threshold.

\section{Sensitivity to lQCD Potential}

In Fig. 7 we show the $c-\bar{c} S$-wave scattering amplitude based on the potential fitted directly to the internal energy data of Ref. [25]. We have kept $m_{c}=1.7 \mathrm{GeV}$ for comparison with our previous 

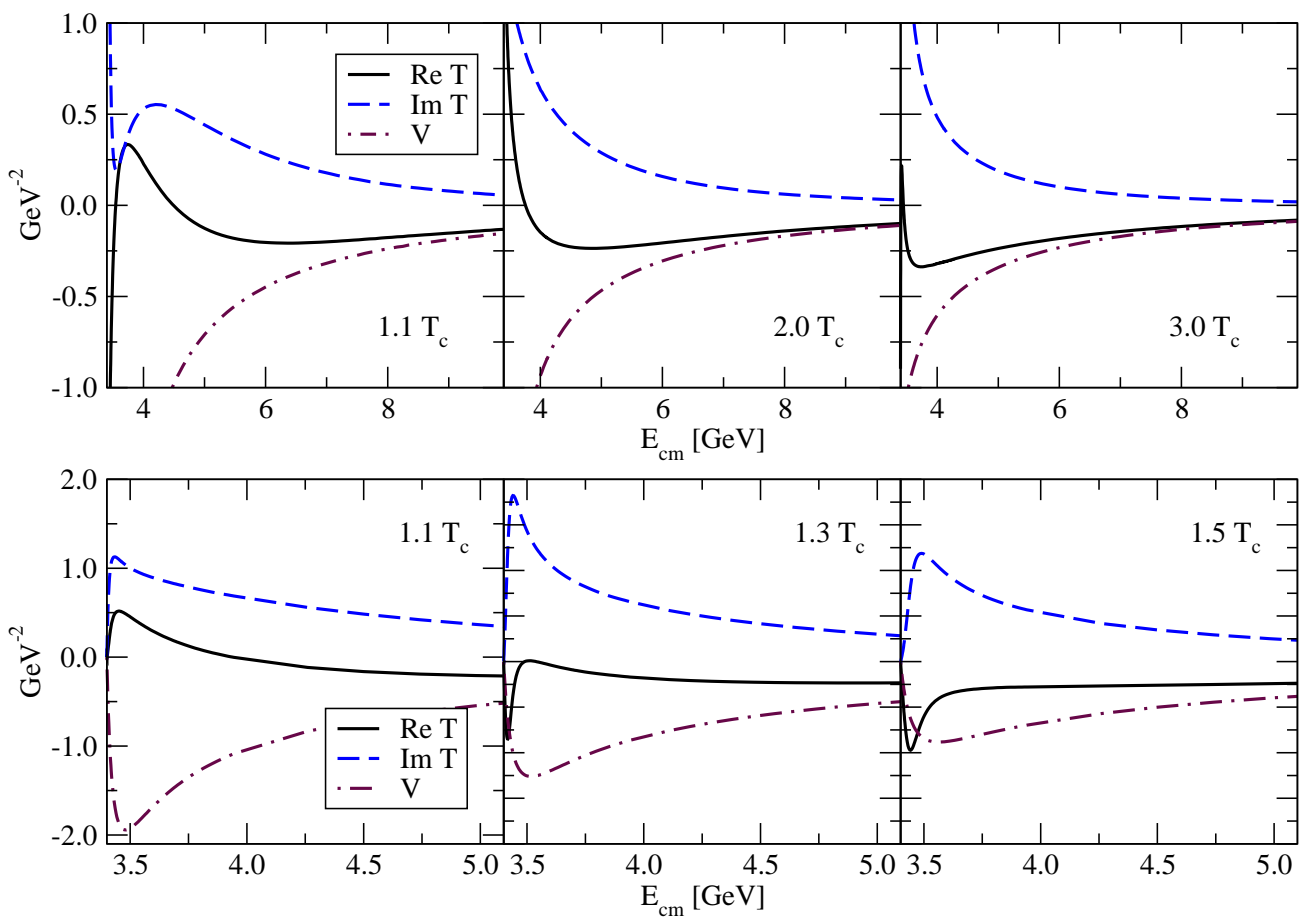

FIG. 5: $c-\bar{c}$ scattering amplitude in $S-$ (up) and $P$-wave (below) above threshold. The Born approximation to the amplitude is also shown (dashed-dotted).
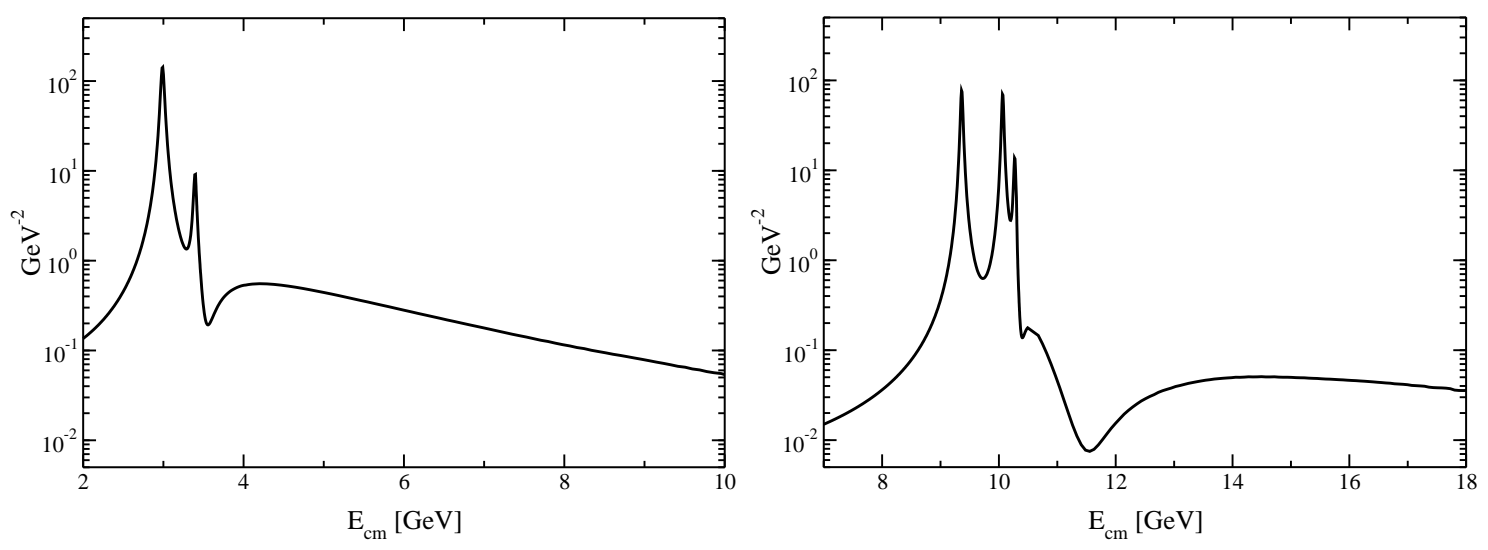

FIG. 6: Imaginary part of the $S$-wave scattering amplitude including bound and scattering parts of the spectrum (left, charmonium; right, bottomonium) at $T=1.1 T_{c}$.

results. The $T$-matrix exhibits two bound states with a stronger binding, as to be expected from the potential comparison in Fig. 1. With the same bare quark mass, the ground state is located at a much lower energy, $E \approx 2.2 \mathrm{GeV}$, translating into a binding energy of about $1.2 \mathrm{GeV}$, in agreement with the results of Refs. [8, 29] within a Schrödinger equation using the same potential. As the temperature increases, the bound states rapidly shift to higher energies, reflecting the rapid reduction of the potential strength at low temperatures. This trend slows down beyond $2 T_{c}$, and the ground state eventually dissolves at $T \simeq 2.5 T_{c}$. The large binding within this potential requires appreciable bare quark masses $\left(m_{c} \sim 2 \mathrm{GeV}\right)$ in order to reproduce the nominal position of the charmonium ground state in the vacuum. The strong attraction is presumably related to the entropy contribution to the $Q-\bar{Q}$ free energy at large distances (cf. Figs. 3 and 4 in Ref. [25]), which 
peaks at $T_{c}$ and decreases steeply with temperature. The large-distance limit of the internal energy, $U_{1}^{\infty}$, which inherits this behavior, is subtracted to generate the $Q-\bar{Q}$ potential, cf. Eq. (10). As mentioned in Sec. IIIA, $U_{1}^{\infty}$ might be interpreted as a contribution to the in-medium quark mass, $m_{c}^{*}(T)=m_{c}+U_{1}^{\infty}(T) / 2$, i.e., a quark selfenergy contribution. However, if no further $r$-dependence (or momentum dependence) is considered, the simple subtraction of $U_{1}^{\infty}$ from the internal energy distorts the normalization of the potential at short distances, where it should be described by perturbative QCD (one-gluon exchange). A more detailed investigation of these interplays will be carried out in future work.

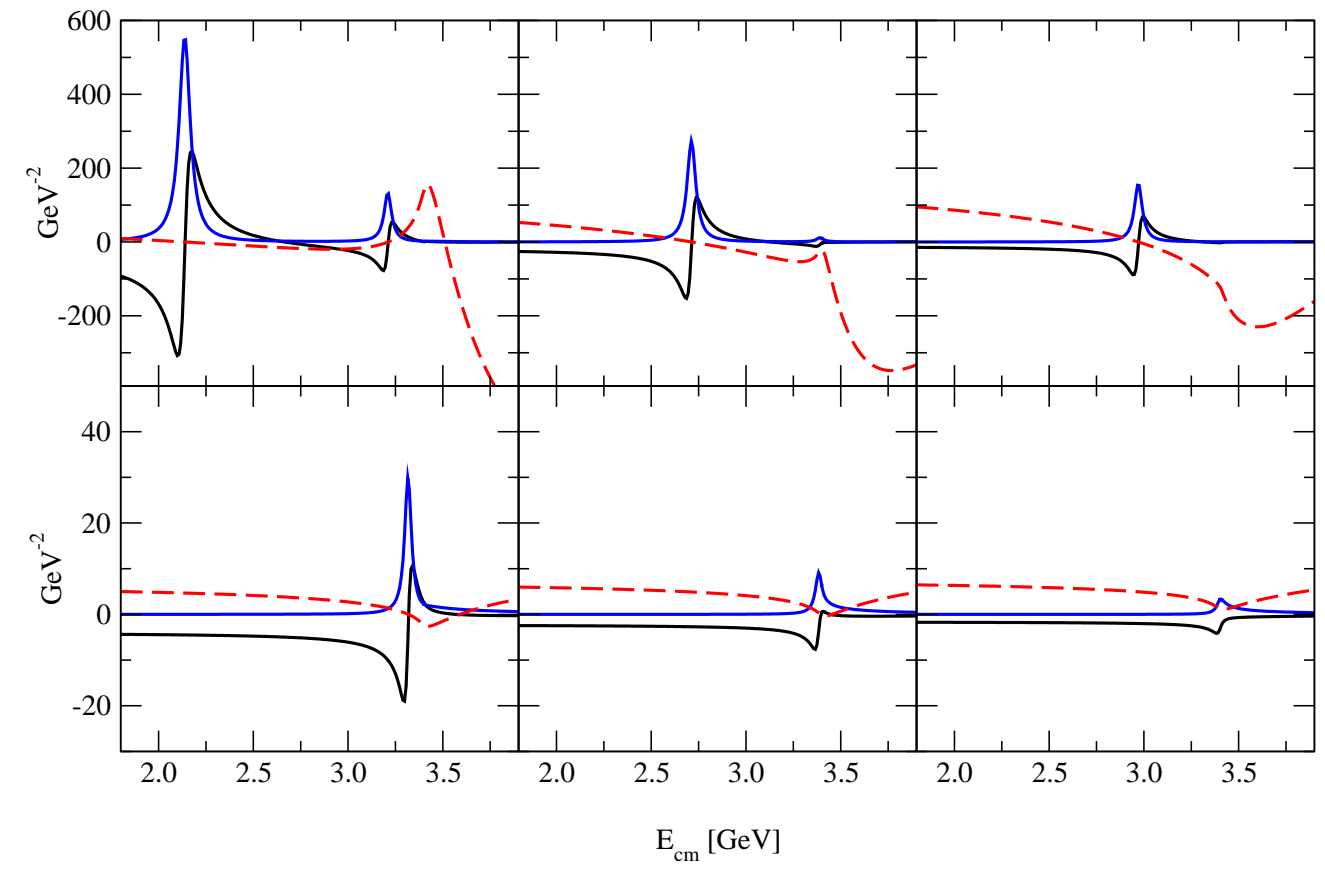

FIG. 7: Same as in Fig. 2 for the potential derived from the lQCD internal energy of Ref. 25]. From left to right and up to down the temperatures are $(1.10,1.15,1.20,1.50,2.00,2.50) T_{c}$.

\section{QUARKONIUM SPECTRAL FUNCTIONS AND EUCLIDEAN-TIME CORRELA- TORS}

\section{A. Spectral Function}

The $T$-matrix formalism used above can be directly applied to evaluate mesonic spectral functions for the different quarkonium channels. The spectral functions encode the information on both the bound and scattering states in the continuum $\left(E>E_{t h}\right)$, similar to the $T$-matrix. Moreover, they allow for a quantitative connection between the present approach and Euclidean-time correlation functions, which have been calculated in lattice QCD with rather high precision [3, 5]. Such a comparison has recently been conducted in Ref. [9] where the heavy-quark interaction in the QGP has been studied by solving the bound-state problem using a Schrödinger equation with either a screened Cornell-type potential or 1QCD-based internal energies (similar to the present work). The quarkonia spectral functions were then composed of $\delta$-function like bound states with weights 
determined by the decay constant of the state and a continuum assuming free quark propagation with a threshold behavior taken from perturbative QCD [30, 31],

$$
\sigma(\omega, T)=\sum_{i} 2 M_{i} F_{i}^{2} \delta\left(\omega^{2}-M_{i}^{2}\right)+\frac{3}{8 \pi^{2}} \omega^{2} f\left(\omega, E_{t h}\right) \Theta\left(\omega-E_{t h}\right) .
$$

The decay constants are related to the (derivative of the) radial wave function at the origin for $S-$ $(P-)$ wave states [32], while the functional form of the continuum threshold, given by $f\left(\omega, E_{t h}\right)$, depends on the specific channel (pseudo-/scalar, axial-/vector) [30, 31]. The threshold energies were set to $E_{t h}^{c \bar{c}}=4.5 \mathrm{GeV}$ and $E_{t h}^{b b}=11 \mathrm{GeV}$, based on the phenomenological observation that no narrow mesonic resonances appear in the spectrum beyond this energies. The resulting correlation functions qualitatively reproduced the features observed in IQCD for the scalar channel $\left(\chi_{c, b}\right)$, whereas sizable discrepancies were found for the pseudoscalar and vector channels $\left(\eta_{c, b}\right.$ and $J / \psi$, $\Upsilon)$.

In the present approach, the $Q-\bar{Q}$ system is interacting also above the $Q-\bar{Q}$ threshold with the same potential that generates the bound-state solutions, which, in particular, accounts for the transition between the discrete and the continuum part of the spectrum. Nonperturbative effects play an especially important role when reduced binding energies drive states toward and across the two-particle threshold. The mesonic spectral function is given by the imaginary part of the heavy-quark two-point (current-current) correlation function in momentum space, $G(E, \vec{P})$, as pictorially depicted by its perturbation series in Fig. 8. The correlation function can be calculated from the $T$-matrix by closing the external legs with the appropriate momentum integrations and the corresponding current operator. Schematically, one has

$$
G=G^{0}+G^{0} T G^{0},
$$

where $G^{0}$ is the lowest order correlation function, which represents the uncorrelated $Q-\bar{Q}$ propagator in a given mesonic channel,

$$
G^{0}(E, \vec{P}=\overrightarrow{0} ; T)=i N_{f} N_{c} \int \frac{d^{3} k}{(2 \pi)^{3}} \operatorname{Tr}\left\{\Gamma_{M} \Lambda_{+}(\vec{k}) \Gamma_{M} \Lambda_{-}(-\vec{k})\right\} G_{Q \bar{Q}}(E ; k)\left[1-2 f^{Q}\left(\omega_{k}\right)\right]
$$

with $\Lambda_{ \pm}(\vec{k})=\left(\omega_{k} \gamma^{0}-\vec{k} \vec{\gamma} \pm m_{Q}\right) / 2 m_{Q}$ the positive/negative energy projectors, $\Gamma_{M}=$ $\left(1, \gamma_{5}, \gamma^{\mu}, \gamma^{\mu} \gamma_{5}\right)$ and $N_{f}\left(N_{c}\right)$ the number of flavors (colors). We take $N_{f}=1, N_{c}=3$ as in [9] to ensure the same normalization of the lowest order correlation function. Eq. (14) denotes the finite-temperature result, and we have used the explicit decomposition of the single particle propagator, $S_{Q}$, in terms of energy projectors, which recovers the BbS 3D-reduction scheme used in the calculation of the $T$-matrix.

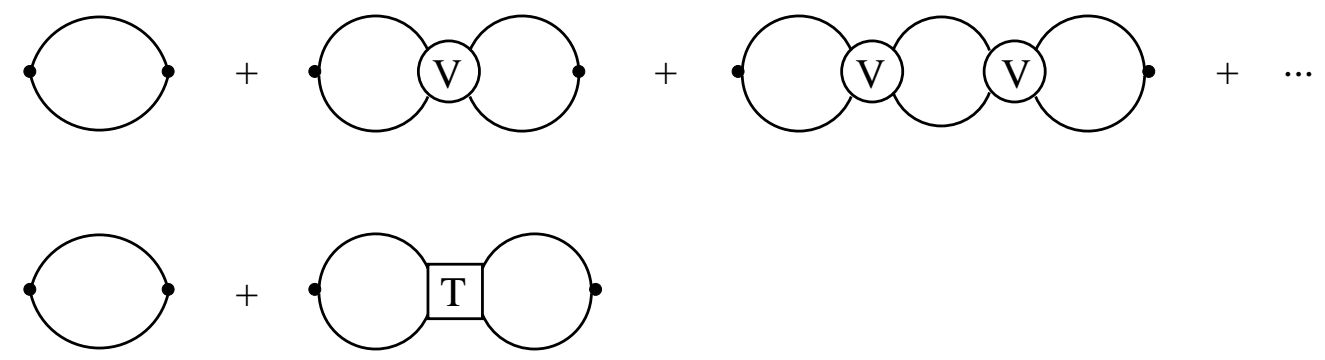

FIG. 8: Diagrammatic representation of the $Q-\bar{Q}$ correlation function. The solid dots represent $\Gamma_{M}$ operators specifying different mesonic channels. 
The $Q-\bar{Q}$ rescattering is encoded in the second (two-loop) term of Eq. (13). A proper connection has to be made between a potential description of the interaction and the relativistic invariant amplitude entering $\Delta G$ (see the Appendix for details). In particular, we have thus far suppressed the tensor structure of $T$ (and $V$ ) in Dirac space. At high energies the $Q-\bar{Q}$ interaction should correspond to perturbative one-gluon exchange, which has a vector structure, whereas at low energies 1QCD finds the potential to be compatible with a scalar structure [33]. In absence of further information, particularly for the intermediate energy regime, we consider both tensor structures alternatively and write for the matrix elements $T_{D}=\bar{u} \tilde{\Gamma} u T \bar{v} \tilde{\Gamma} v$, with $\tilde{\Gamma}=1, \gamma^{\nu}$ and $u(v)$ the positive (negative) energy Dirac spinors. This leads to the following traces to be evaluated in Eq. (13):

$$
\operatorname{Tr}\left(\Gamma_{M}, \tilde{\Gamma}\right)=\operatorname{Tr}\left\{\Lambda_{+}(\vec{k}) \Gamma_{M} \Lambda_{-}(-\vec{k}) \tilde{\Gamma} \Lambda_{-}\left(-\vec{k}^{\prime}\right) \Gamma_{M} \Lambda_{+}\left(\vec{k}^{\prime}\right) \tilde{\Gamma}\right\}
$$

It turns out that they can be written in a partial wave expansion as performed for the $T$-matrix,

$$
\operatorname{Tr}\left(\Gamma_{M}, \tilde{\Gamma}\right)=a_{0}^{\Gamma_{M}, \tilde{\Gamma}}\left(k, k^{\prime}\right) P_{0}\left(\cos \theta_{k k^{\prime}}\right)+a_{1}^{\Gamma_{M}, \tilde{\Gamma}}\left(k, k^{\prime}\right) P_{1}\left(\cos \theta_{k k^{\prime}}\right)+a_{2}^{\Gamma_{M}, \tilde{\Gamma}}\left(k, k^{\prime}\right) P_{2}\left(\cos \theta_{k k^{\prime}}\right),
$$

so that all angular integrations can be done analytically by using the orthogonality of the Legendre polynomials. We thus have

$$
\begin{aligned}
\Delta G(E ; T) & =N_{f} N_{c} \frac{1}{8 \pi^{4}} \int d k k^{2} G_{Q \bar{Q}}(E ; k)\left[1-2 f^{Q}\left(\omega_{k}\right)\right] \\
& \times \int d k^{\prime} k^{\prime 2} G_{Q \bar{Q}}\left(E ; k^{\prime}\right)\left[1-2 f^{Q}\left(\omega_{k^{\prime}}\right)\right] \mathcal{T}\left(\Gamma_{M}, \tilde{\Gamma} ; E ; k, k^{\prime}\right),
\end{aligned}
$$

with the kernel $\mathcal{T}$ given by

$$
\begin{aligned}
\mathcal{T}\left(\Gamma_{M}, \tilde{\Gamma} ; E ; k, k^{\prime}\right) & \equiv \int d\left(\cos \theta_{k k^{\prime}}\right) \operatorname{Tr}\left(\Gamma_{M}, \tilde{\Gamma} ; k, k^{\prime}, \theta_{k k^{\prime}}\right) T\left(E ; \vec{k}, \vec{k}^{\prime}\right) \\
& =8 \pi\left[a_{0}\left(k, k^{\prime}\right) T_{0}\left(E ; k, k^{\prime}\right)+a_{1}\left(k, k^{\prime}\right) T_{1}\left(E ; k, k^{\prime}\right)\right],
\end{aligned}
$$

and $a_{l}$ coefficients as tabulated in Table II. We note that for a given channel, e.g. pseudoscalar, in principle both the $S$ - and $P$-wave components of the $T$-matrix contribute to the correlation function, whereas the usual spectroscopic (nonrelativistic) characterization of quarkonium states is based on orbital angular momentum quantum numbers ( $L S$ scheme). The (undesired) mixing of $S$ - and $P$-wave components in the correlation function is related to the use of the $J M$ (helicity) basis of the $Q-\bar{Q}$ spectrum at high energies, in which a different partial wave decomposition of the $T$-matrix follows. However, for the scalar and pseudoscalar channels the coefficient in Table II corresponding to the "natural" partial wave is leading in the non-relativistic (heavy-quark) expansion, whereas the other one, introducing an admixture of the "unnatural" partial wave, is of higher order, cf. Table III] For simplicity, we shall work with the non-relativistic approximation for the $a_{l}$ coefficients, which filters the appropriate partial wave for the scalar and pseudoscalar channels (and for consistency with the spin-averaged nature of the interaction potential we shall consider pseudoscalar/vector, scalar/axialvector degeneracy for the correlation functions as done for the $T$-matrix $)^{5}$. We comment below on the accuracy of this approximation.

\footnotetext{
${ }^{5}$ Note that at this level of approximation the distinction between a scalar- or vector-like structure for $T$ is immaterial since both have the same heavy-quark limit for the $a_{l}$ coefficients (modulo global signs).
} 


\begin{tabular}{|c||c|c|}
$\mid \Gamma_{M}, \tilde{\Gamma}$ & $a_{0}\left(k, k^{\prime}\right)$ & $a_{1}\left(k, k^{\prime}\right)$ \\
\hline \hline $\mathrm{S}, \mathrm{S}$ & $\frac{k^{2} k^{\prime 2}}{m_{q}^{4}}$ & $-\left(\frac{\omega_{k} \omega_{k^{\prime}}}{m_{q}^{2}}+1\right) \frac{k k^{\prime}}{m_{q}^{2}}$ \\
\hline $\mathrm{S}, \mathrm{V}$ & $4 \frac{k^{2} k^{\prime 2}}{m_{q}^{4}}$ & $2 \frac{k k^{\prime}}{m_{q}^{2}}$ \\
\hline $\mathrm{PS}, \mathrm{S}$ & $1+\frac{\omega_{k} \omega_{k^{\prime}}}{m_{q}^{2}}+\frac{k^{2}+k^{\prime 2}}{m_{q}^{2}}+\frac{k^{2} k^{\prime 2}}{m_{q}^{4}}$ & $-\frac{\omega_{k} \omega_{k \prime}}{m_{q}^{2}} \frac{k k^{\prime}}{m_{q}^{2}}$ \\
\hline $\mathrm{PS}, \mathrm{V}$ & $-2\left(1+\frac{m_{q}^{2}-\omega_{k} \omega_{k^{\prime}}}{m_{q}^{2}}\right)-4\left(\frac{k^{2}+k^{\prime 2}}{m_{q}^{2}}+\frac{k k^{\prime}}{m_{q}^{2}}\right)$ & 0 \\
\hline $\mathrm{V}, \mathrm{S}$ & $3\left(1+\frac{\omega_{k} \omega_{k^{\prime}}}{m_{q}^{2}}\right)+2 \frac{\omega_{k} \omega_{k^{\prime}}}{m_{q}^{2}}+\frac{4}{3} \frac{k k^{\prime}}{m_{q}^{2}}$ & $-\left(2 \frac{\omega_{k} \omega_{k^{\prime}}}{m_{q}^{2}}+1\right) \frac{k k^{\prime}}{m_{q}^{2}}$ \\
\hline $\mathrm{V}, \mathrm{V}$ & $-6-4 \frac{k^{2}+k^{\prime 2}}{m_{q}^{2}}-\frac{8}{3} \frac{k^{2} k^{\prime 2}}{m_{q}^{4}}$ & $-4\left(1+\frac{\omega_{k} \omega_{k^{\prime}}}{m_{q}^{2}}\right) \frac{k k^{\prime}}{m_{q}^{2}}$ \\
\hline $\mathrm{AV}, \mathrm{S}$ & $-1-\frac{\omega_{k} \omega_{k^{\prime}}}{m_{q}^{2}}-\frac{4}{3} \frac{k^{2} k^{\prime 2}}{m_{q}^{4}}$ & $\left(2 \frac{\omega_{k} \omega_{k^{\prime}}}{m_{q}^{2}}+3\right) \frac{k k^{\prime}}{m_{q}^{2}}$ \\
\hline $\mathrm{AV}, \mathrm{V}$ & $2\left(2 \frac{\omega_{k} \omega_{k^{\prime}}}{m_{q}^{2}}-1-\frac{8}{3} \frac{k^{\prime} k^{\prime 2}}{m_{q}^{4}}\right)$ & $-4 \frac{\omega_{k} \omega_{k^{\prime}}}{m_{q}^{2}} \frac{\omega_{k} \omega_{k^{\prime}}}{m_{q}^{2}}$ \\
\hline
\end{tabular}

TABLE II: $a_{l}$ coefficients in a partial wave basis up to $L=1$.

\begin{tabular}{|c||c|c|}
$\mid \Gamma_{M}, \tilde{\Gamma}$ & $a_{0}\left(k, k^{\prime}\right)$ & $a_{1}\left(k, k^{\prime}\right)$ \\
\hline \hline $\mathrm{S}, \mathrm{S}$ & $\mathcal{O}\left(k^{2} / m_{q}^{2}\right)$ & $-2 \frac{k k^{\prime}}{m_{q}^{2}}+\mathcal{O}\left(k^{2} / m_{q}^{2}\right)$ \\
\hline $\mathrm{S}, \mathrm{V}$ & $\mathcal{O}\left(k^{2} / m_{q}^{2}\right)$ & $2 \frac{k k^{\prime}}{m_{q}^{2}}$ \\
\hline $\mathrm{PS}, \mathrm{S}$ & $2+\mathcal{O}\left(k^{2} / m_{q}^{2}\right)$ & $\mathcal{O}\left(k / m_{q}\right)$ \\
\hline $\mathrm{PS}, \mathrm{V}$ & $-2+\mathcal{O}\left(k^{2} / m_{q}^{2}\right)$ & 0 \\
\hline
\end{tabular}

TABLE III: Lowest order of $a_{l}$ coefficients in a $(1 / m)$ expansion.

\section{B. Euclidean-Time Correlation Functions}

The Euclidean-time correlation function is defined as the thermal mesonic two-point correlation function in a mixed Euclidean-time-momentum representation [30, 31, 34]. It can be expressed in a spectral representation as an integral transformation of the mesonic spectral function (here $\vec{P}=\overrightarrow{0})$,

$$
G(\tau, T)=\int_{0}^{\infty} d \omega \sigma(\omega, T) \mathcal{K}(\tau, \omega, T)
$$

where the kernel of the transformation,

$$
\mathcal{K}(\tau, \omega, T)=\frac{\cosh [\omega(\tau-\beta / 2)]}{\sinh (\omega \beta / 2)},
$$

is symmetric with respect to $\tau=\beta / 2$ (and $\tau \in[0, \beta]$ ). The Euclidean-time correlation function scans the full spectrum of the system. In particular, for $\tau \rightarrow 0$ the kernel decreases rather slowly with energy and thus the correlation function is dominated by contributions of the $Q-\bar{Q}$ continuum. On the other hand, for $\tau \rightarrow \beta / 2$, the kernel exhibits the maximal decrease, so that the correlation function becomes mostly sensitive to the contribution from the low-energy region of the spectrum, in particular the bound states. To isolate the medium effects on the mesonic spectral function from the temperature dependence introduced by the kernel it has been proposed [3, 5] to normalize the correlation function at a given temperature to a so-called "reconstructed" correlation function,

$$
G_{r}(\tau, T)=\int_{0}^{\infty} d \omega \sigma(\omega, T=0) \mathcal{K}(\tau, \omega, T)
$$

which is obtained by replacing $\sigma(\omega, T)$ by a reference spectral function (for instance the vacuum spectral function) and transformed with the same finite-temperature integral kernel. As in Ref. [9], 
we will first assume $\sigma(\omega, T=0)$ of the form in Eq. (12) with the vacuum input for the boundstate part and a shape function $f\left(\omega, E_{t h}\right)$ given by the perturbative QCD continuum. For the open-charm (bottom) threshold, we consider $E_{t h}^{c \bar{c}(b \bar{b})}=2 M_{D(B)}=3.74(10.56) \mathrm{GeV}$, but also check the sensitivity to changes in the pertinent free open heavy-flavor meson thresholds by using $E_{t h}^{c \bar{c}(b \bar{b})}=4.5(11) \mathrm{GeV}$ as in Ref. [9]. As we shall see, the use of a simplified spectral function as in Eq. (12) may introduce spurious features in the normalized correlation function which could mask the actual effect of the medium-modified $Q-\bar{Q}$ interaction. In order to enable a more direct comparison to lQCD evaluations, we will also normalize our results to actual spectral functions calculated in our approach.

It is clear from Eq. (19) that the full energy regime of the spectral function figures into the calculation of the Euclidean-time correlation function. The approximations introduced in Sec. IVA are expected to be reliable up to energies above the $Q-\bar{Q}$ threshold, where non-perturbative effects from the $Q-\bar{Q}$ interaction prevail, as is already manifest from non-trivial structure in the $T$-matrix. For higher energies in the continuum region we do not expect these approximations to hold. However, the high energy part of the continuum should be only relevant for $\tau \rightarrow 0$, where the normalized correlation function approaches 1 and is not sensitive to the evolution of the quarkonia states with the temperature.

\section{Numerical Results}

\section{Constant Heavy-Quark Mass and Small Width}

Following our studies of the $T$-matrices, we will first investigate mesonic spectral functions and normalized correlation functions for a constant quark mass, and therefore the continuum threshold is not dependent on the temperature. The $S$-wave charmonium spectral function is shown in the left panel of Fig. 9 for several temperatures, together with the uncorrelated (perturbative) twoparticle continuum, Eq. (14). As expected, the spectral function exhibits the same charmonium bound states as found in the $T$-matrix, as well as their evolution to higher energies as the temperature is increased. At all temperatures, the (non-perturbative) rescattering of the $Q-\bar{Q}$ system dynamically generates a substantial enhancement of strength above the $c-\bar{c}$ threshold relative to the uncorrelated two-particle continuum (at $T=1.1 T_{c}$ the remnant of the first excited state $(\psi(2 S)$ ) is still visible). This important effect reflects the $Q-\bar{Q}$ correlations already found for the $T$-matrix above threshold (especially when a bound state passes into the continuum).

The corresponding normalized Euclidean correlation function for the same set of temperatures is displayed in the right panel of Fig. 9. The normalized correlator converges to unity at $\tau \rightarrow 0$, which reconfirms the correct normalization of the continuum part of the spectrum (it is also symmetric with respect to $\beta / 2$ ). At low $\tau$, where the integral in Eq. (19) is mostly dominated by the continuum region, the normalized correlator moderately increases, reaches a maximum, and then rapidly drops for $\tau$ approaching $\beta / 2$, indicating a loss of strength of the finite temperature correlator relative to the zero-temperature one in the low-energy part of the spectrum. The temperature evolution of the correlation function is a combined result of a decrease in binding energy of the bound states and the contribution of the non-perturbative continuum. The sizable drop at large $\tau$ is in qualitative agreement with the lQCD charmonium $S$-wave correlator [3]. The latter, though, exhibits a smaller reduction and a weaker $\tau$-dependence, showing appreciable deviations from unity only beyond $T=1.5 T_{c}$. 

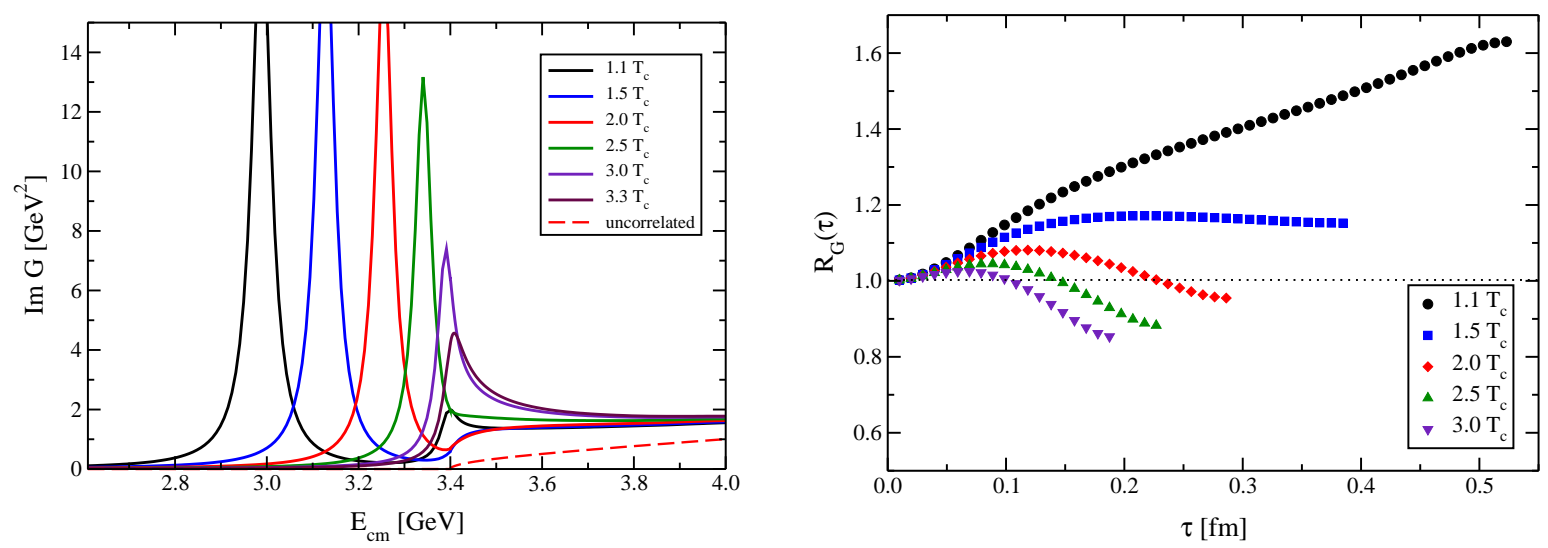

FIG. 9: Left panel: Imaginary part of the correlated two-particle propagator (Green's function) for $c-\bar{c}$ $S$-wave scattering at several temperatures and constant quark mass, $m_{c}=1.7 \mathrm{GeV}$. The imaginary part of the uncorrelated propagator (dashed line) is also shown for reference. Right panel: Corresponding normalized mesonic correlation functions.

The charmonium spectral function and normalized correlator for $P$-wave scattering is displayed in Fig. 10. As was already discussed in Sec. IIIB, we only find a single bound state $\left(\chi_{c}\right)$ just below the threshold, which rapidly melts into the continuum as the temperature increases. Consequently, a sizable threshold enhancement effect is observed. Despite the fast melting of the $P$-wave state, the correlation function steeply rises in the low- $\tau$ regime, due to (i) the contribution from the non-perturbative rescattering above threshold, and (ii) a larger threshold energy in the schematic vacuum spectral function entering the "reconstructed" correlator. For $\tau \rightarrow \beta / 2$, the correlator levels off well above unity, due to the absence of an energy gap between the $P$-wave state and the continuum, which renders the (enhanced) continuum contribution to the correlator dominant even for $\tau \rightarrow \beta / 2$. The main features of our results at a given temperature are qualitatively in line with the lQCD $P$-wave correlators [3]; however, the temperature dependence is not: our correlators attenuate with temperature whereas the 1QCD correlator increases. This appears to be a rather direct indication that the in-medium $c-\bar{c}$ threshold is lowered with increasing temperature.

While our results are qualitatively similar to those of Ref. [9], the following observations are in order. In Ref. 9], the increase of the correlator at low and intermediate $\tau$ is induced by the temperature-dependent decrease of the continuum threshold. Thus far, we have not considered the temperature effect on the $Q-\bar{Q}$ threshold. However, the non-perturbative enhancement in the spectral function around threshold and above, which is not included in Ref. 9], turns out to be essential for a quantitative assessment of the quarkonium correlation (and spectral) functions, especially when the energy gap between the discrete and continuum parts of the spectrum is small or absent [26, 35, 36]. Nevertheless, our results leave room for a lowering of the $Q-\bar{Q}$ threshold energy, since a downward shift of strength in the spectral function would improve (i) on the large$\tau$ - decrease in the $S$-wave correlator, and (ii) on the temperature dependence in the $P$-wave correlator, as discussed below.

The bottomonium spectral and correlation functions follow a similar pattern as for the charmonium system. The results for $S$-wave scattering are displayed in Fig. 11, At large Euclidean time, the correlator moderately decreases with temperature as the two excited bottomonia dissappear into the continuum. The contribution of the $\Upsilon(1 S)$ state, which survives up to rather high temperatures, makes the correlator fall more slowly than in the charmonium case. We find a 

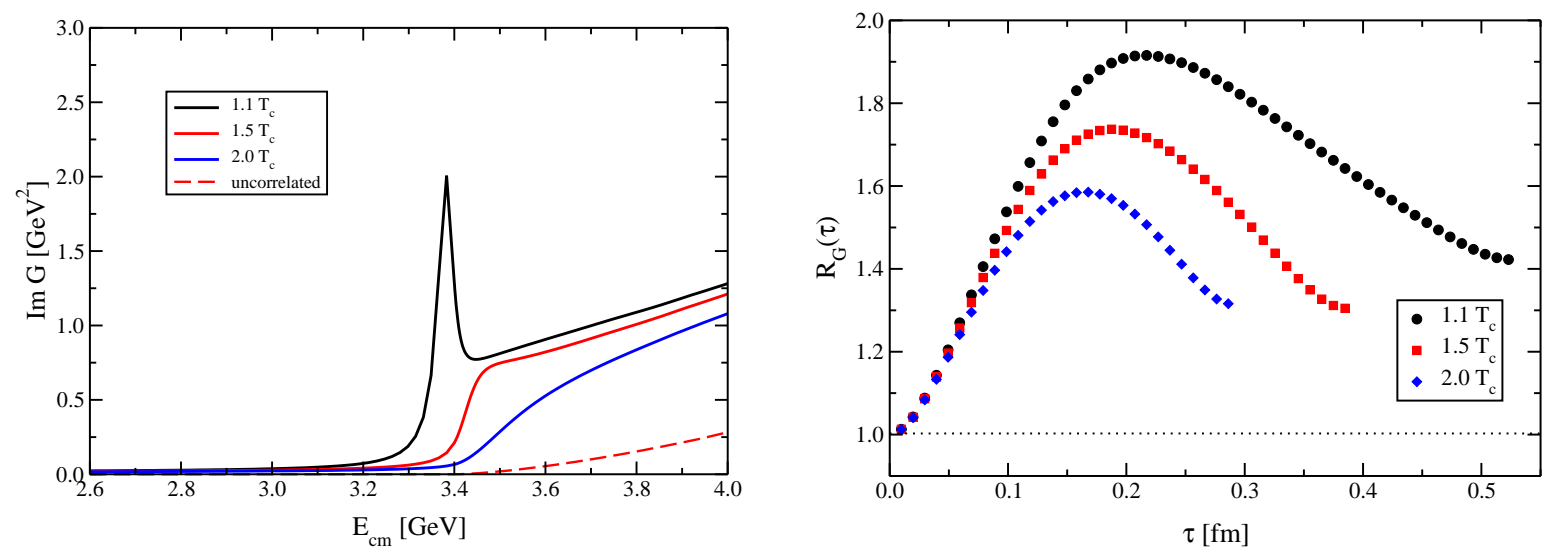

FIG. 10: Same as in Fig. 9 for $c-\bar{c} P$-wave scattering.

similar enhancement at low $\tau$, partly due to the continuum contribution with respect to the zero temperature case, which becomes more relevant as the bound-state binding energies decrease with temperature.
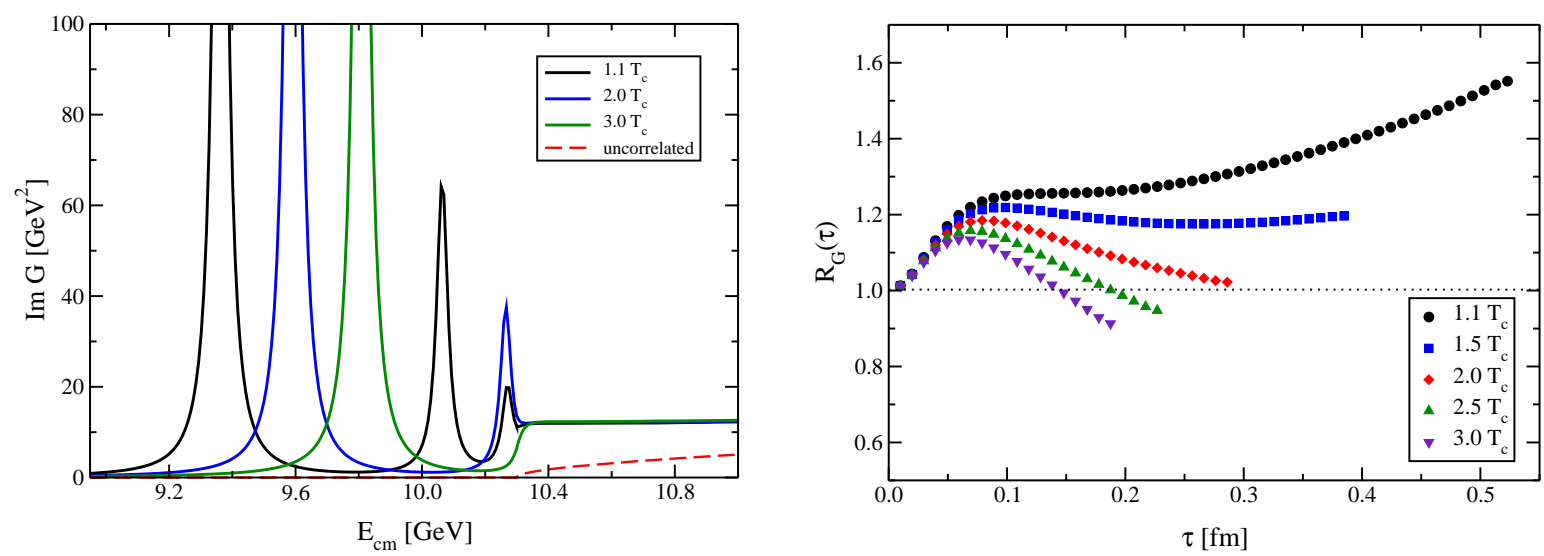

FIG. 11: Same as in Fig. 9 for $b-\bar{b} S$-wave scattering.

The $P$-wave bottomonium correlator, Fig. [12, shows a large enhancement at all $\tau$, even larger than that of the $P$-wave charmonium correlator. A similar enhancement is observed in the scalar bottomonium correlator from IQCD [5]. As the temperature increases, the two bound states gradually move to higher energies and the correlator is notably attenuated. Again, as the ground state approaches the continuum the nonperturbative threshold strength in the spectral function is the decisive source of the remaining correlator enhancement. This reiterates the point that a comprehensive description of the Euclidean-time correlators should account for both the bound state evolution and $Q-\bar{Q}$ correlations above threshold.

\section{Sensitivity to the Reconstructed Correlator}

Although our results thus far roughly reproduce some of the trends of the normalized correlators from lQCD, the $S$-wave correlator exhibits a marked $\tau$ dependence which is inconsistent with lQCD 

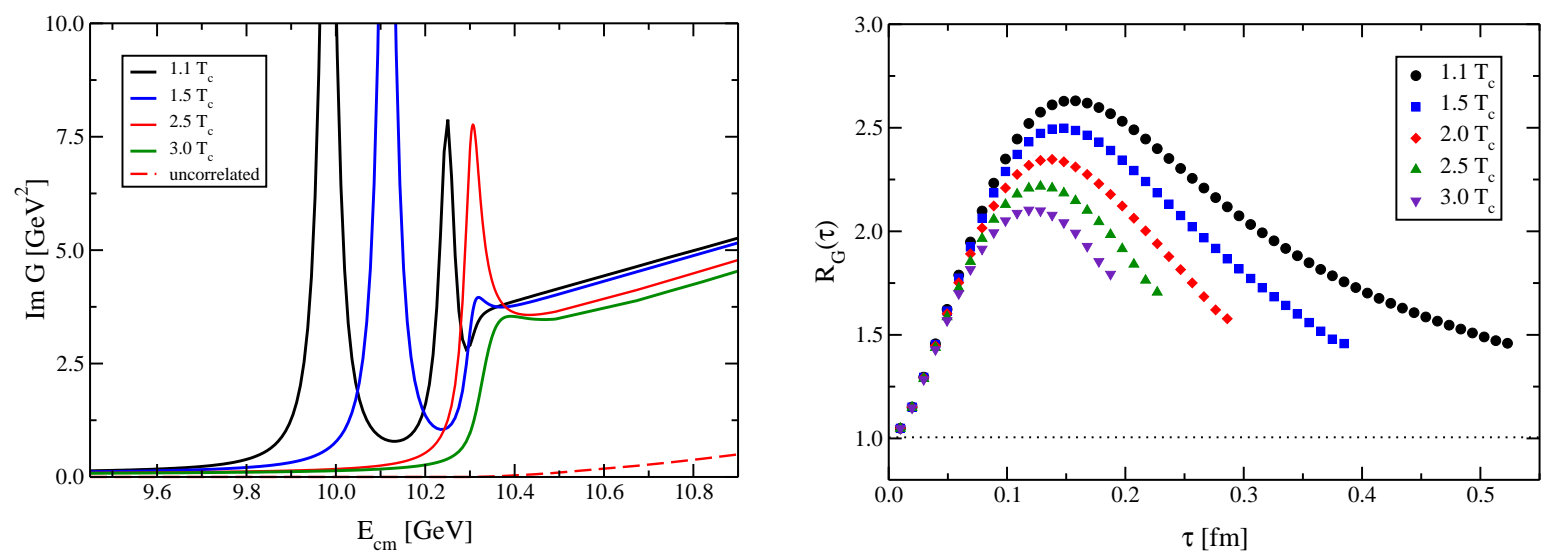

FIG. 12: Same as in Fig. 9 for $b-\bar{b} P$-wave scattering.
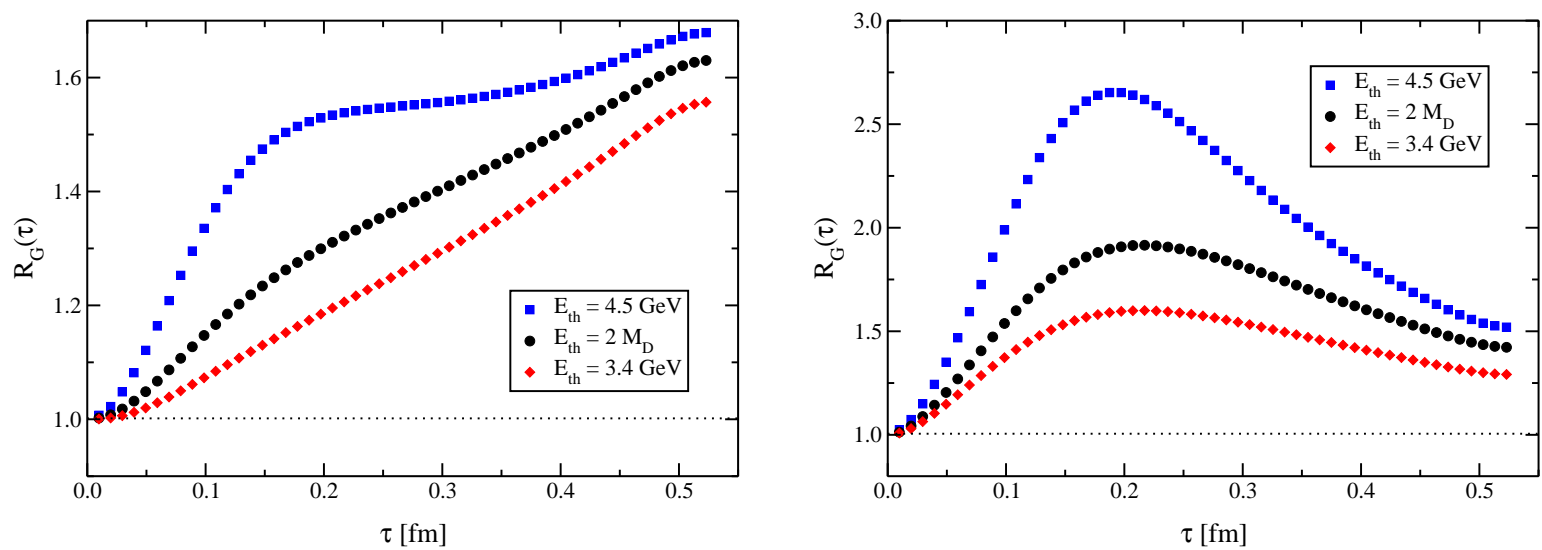

FIG. 13: Normalized correlation function for $c-\bar{c} S$-wave (left) and $P$-wave (right) scattering at $T=$ $1.1 T_{c}$. As indicated in the legend, three different continuum threshold energies have been used in the zero temperature spectral function: $E_{t h}^{c \bar{c}}=4.5 \mathrm{GeV}$ as in Ref. [9], the open-charm threshold, and $E_{t h}^{c \bar{c}}=2 m_{c}$ with $m_{c}=1.7 \mathrm{GeV}$.

results. In particular, in the low- and intermediate- $\tau$ regime, our $S$-wave correlators significantly increase even for temperatures just above $T_{c}$, while in LCD they are essentially unmodified until about $1.5 T_{c}$. As discussed above, one reason for this rise is the threshold mismatch between the finite-temperature spectral function and the vacuum one, which is modeled according to Eq. (12). We therefore investigate two alternative assumptions for the reconstructed correlator (which is used for normalization).

First, we change the continuum energy threshold of the vacuum spectral function by varying the above values in the range $E_{t h}^{c \bar{c}(b \bar{b})}=3.4-4.5(10.3-11.0) \mathrm{GeV}$. In Fig. 13 we compare the resulting charmonium correlation function to our earlier result (right panels in Figs. 9 and 10) at the lowest temperature, $T=1.1 T_{c}$. Not surprisingly, for both $S$ - and $P$-wave scattering the low- $\tau$ rise of the amplitude is strongly reduced as the threshold energy is shifted to lower values. At large $\tau$, the $S$-wave correlator changes only slightly, as expected since the discrete (low-energy) part of the spectrum in the reference spectral function is unchanged. However we note comparably larger changes of the $P$-wave correlator also at large $\tau$, since the small binding of the $\chi_{c}$ makes the correlator sensitive to the continuum contribution in the entire $\tau$ domain. 


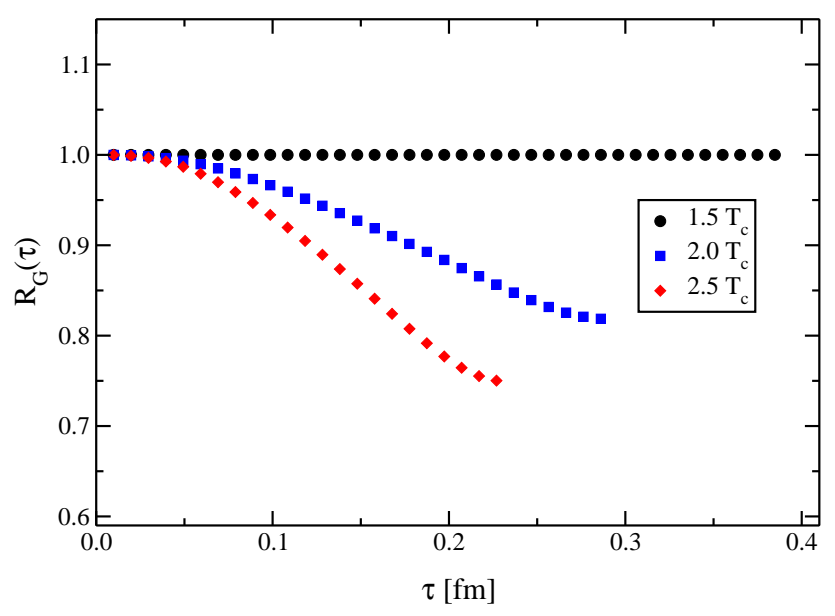

FIG. 14: Same as in Fig. 9 but using the in-medium correlator at $T=1.5 T_{c}$ as the "reconstructed" correlator to normalize the plotted ratio.

Second, we make use of the observation that in the $S$-wave channel the (normalized) lQCD correlators are essentially unchanged compared to the reconstructed correlator up to temperatures of about $1.5 T_{c}$. In order to remove ambiguities from the use of a simplified reference spectral function in the reconstructed correlator, we use instead our calculated in-medium spectral function at $1.5 T_{c}$, which incorporates the simultaneous description of bound and scattering states. Thus, by construction, the correlator ratio at $1.5 T_{c}$ is identically one, consistent with lQCD. The nontrivial result is now the further temperature evolution, as displayed in Fig. 14. It turns out that the new normalized correlator shows a substantially weaker $\tau$ dependence, decreasing with increasing temperature in a better agreement with the lQCD correlator.

To summarize this section, we have shown that the correlator ratios are quite sensitive to the underlying "reconstructed" correlators used for normalization, translating into appreciable variations in the absolute magnitude of the normalized correlators (especially in the low- $\tau$ regime). We thus find that current discrepancies between lQCD correlation functions and potential-model approaches (such as in the previous Section, as well as in earlier works 9]) do not necessarily invalidate the latter, especially if one recalls the present additional uncertainty of how to define a $Q-\bar{Q}$ potential from the lattice free/internal energy [7, 26].

\section{In-Medium Heavy-Quark Masses and Widths}

We finally consider the effect of in-medium properties of the interacting heavy quarks. First, we incorporate an effective in-medium quark mass as extracted from the large-distance plateau of the internal energy, $m_{Q}^{*}(T)=m_{Q}+U_{1}^{\infty}(T) / 2$, and we neglect a possible momentum dependence of this correction. We note that in our approach an effective reduction of the heavy-quark mass does not only modify the $Q-\bar{Q}$ threshold energy $\left(E_{t h}=2 \omega_{q=0}=2 m_{Q}^{*}\right)$, but also figures into the two-particle propagator as a selfenergy contribution and therefore is iterated in the scattering equation. We again adjust the bare quark mass to recover the vacuum mass of the quarkonium ground state at $1.1 T_{c}$. The charmonium spectral function for $S$-wave scattering (left panel of Fig. 15) shows a sizable downward shift of the bound state strength, which for higher temperatures is compensated by the reduction in the binding energy. As expected, the dissociation temperature of the ground state is smaller, $T_{\text {diss }} \approx 2.5 T_{c}$, as compared to the calculation with a fixed quark 
mass. The Euclidean correlator (right panel in Fig. 15), which we normalize to the $1.5 T_{c}$ spectral function as discussed in the previous section, has a rather mild temperature dependence, with an attenuation of the order of $10-15 \%$, in a closer agreement with the $S$-wave lQCD correlator. Thus, for $T \geq 1.5 T_{c}$, the $T$-dependent (decreasing) threshold improves the agreement with the lattice calculations. On the other hand, a strong $T$ dependence as dictated by the large-distance behavior of the internal energy at $T \leq 1.5 T_{c}$ would not be supported the 1QCD correlator (a recent analysis of charmonium properties in quenched $1 \mathrm{QCD}$ shows a temperature dependent charmonium mass reduction of about $6 \%$ at $2 T_{c}$ [37]). For more stringent conclusions the present uncertainties in the extraction of the heavy-quark potential, as well as the assumption of a 3-momentum independent mass correction (which upsets the perturbative normalization of the potentials at short distances and therefore represents an upper estimate of the in-medium mass effect), need to be scrutinized.
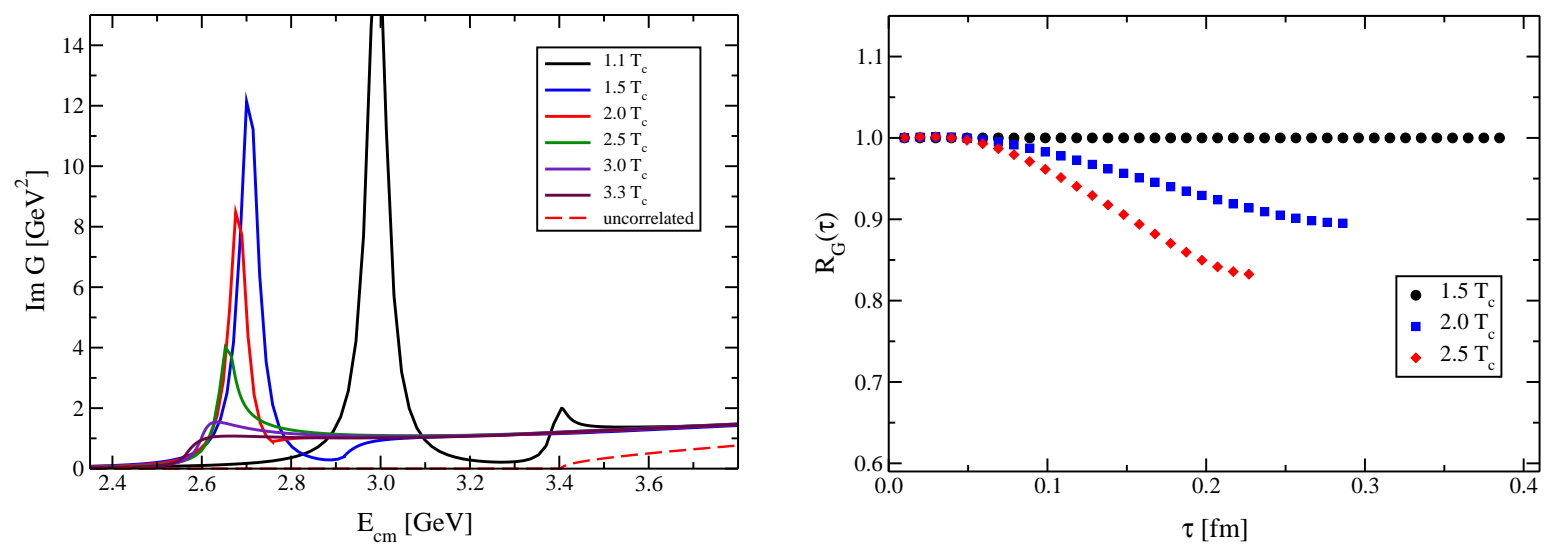

FIG. 15: Left: Imaginary part of the correlated two-particle propagator (Green's function) for $c-\bar{c} S-$ wave scattering at several temperatures. The temperature dependent effective quark mass, $m_{q}^{*}(T)$, has been used in the calculation. Right: Corresponding normalized mesonic correlation function at several temperatures.

We furthermore study the impact of finite quarkonium widths, which are an essential ingredient in the phenomenology of charm and bottom in heavy-ion collisions. As suggested by recent analysis of heavy-quark diffusion in a QGP [20, 21], as well as parton-induced break-up reactions of charmonia [11], their widths are expected to be of the order of $100 \mathrm{MeV}$ at temperatures around $1.5 T_{c}$. We can easily incorporate such effects by dressing the charm quarks with an imaginary selfenergy in the two-particle propagator, Eq. (2). The results for the $S$-wave charmonium correlation function in the fixed quark mass approach are shown in Fig. 16, where a charm width of $50 \mathrm{MeV}$ (generating charmonium widths of $\sim 100 \mathrm{MeV}$ ) has been used, in comparison with the narrow-width limit. At $2 T_{c}$ the Euclidean correlator is modified by only a few percent. Especially in view of other current uncertainties, the correlators are rather insensitive to phenomenologically relevant magnitudes of the quarkonium decay widths; this may after all not be surprising since $\Gamma_{\psi}$ is very much smaller than the charmonium mass, and also appreciably smaller than the typical temperature. 


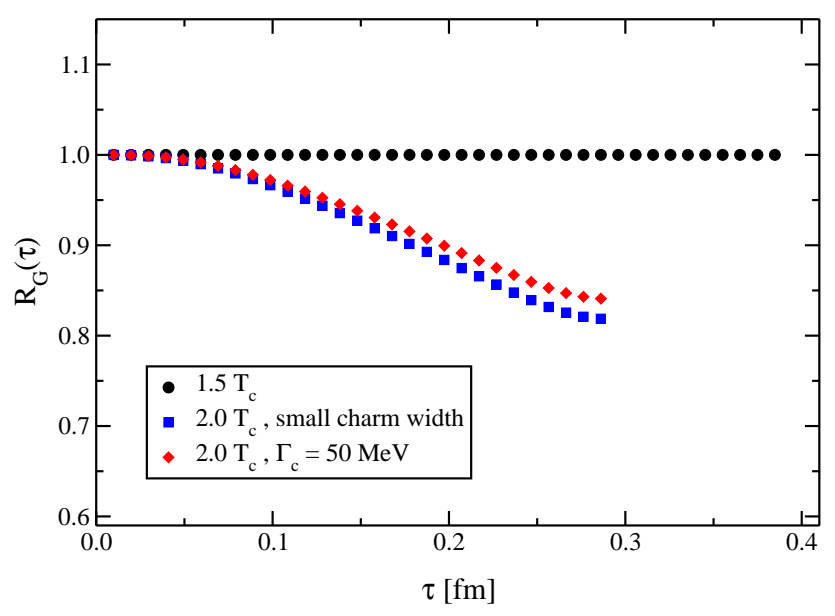

FIG. 16: $S$-wave charmonium correlator with a charm width of $50 \mathrm{MeV}$, as compared to the narrow quark calculation.

\section{CONCLUSIONS AND OUTLOOK}

In the present article we have evaluated spectral properties of heavy quark-antiquark interactions (charm and bottom) in the Quark-Gluon Plasma within a $T$-matrix approach which allows for a comprehensive treatment of bound and scattering states. The basic interaction was taken to be a two-body potential which, following earlier works, has been identified with the heavy-quark internal energy evaluated in thermal lattice QCD. The finite-temperature $T$-matrices reconfirmed the survival of ground-state $\left(S\right.$-wave) bound states for temperatures well above $T_{c}$ as found in earlier calculations, up to $\sim 2.8 T_{c}\left(>3.5 T_{c}\right)$ for $\eta_{c} / J / \psi\left(\eta_{b} / \Upsilon\right)$, as well as for $P$-wave and excited bottomonia. The dissociation mechanism is characterized by a bound state passing through the $Q-\bar{Q}$ threshold, at which point a strong reduction and broadening of the (imaginary part of the) $T$-matrix is observed. Nevertheless, the $Q-\bar{Q}$ system remains strongly correlated in the continuum, indicated by resonant-like structures in the $T$-matrix (albeit at much reduced magnitude) which deviate from the Born approximation up to energies of $1-2 \mathrm{GeV}$ above $Q-\bar{Q}$ threshold.

We have proceeded to calculate $Q-\bar{Q}$ current-current correlation functions, which, in the timelike regime, follow from the $T$-matrix by folding with the $Q-\bar{Q}$ propagator. The imaginary part of the correlation function (spectral function) confirmed the importance of threshold effects in the dissolution mechanisms, resulting in large (nonperturbative) enhancements over the perturbative form of the continuum. These, in turn, give substantial contributions to the Euclidean correlators primarily at large and intermediate time, $\tau$, which cannot be neglected in quantitative comparisons to (and interpretations of) lattice QCD results. Assuming constant heavy-quark masses, some qualitative features of the lQCD correlators (large- $\tau$ decrease in the $S$-waves, overall increase in the $P$-waves) could be reproduced. However, the magnitude of the signal in the $S$-wave channels, as well as the temperature dependence in the $P$-wave channels, were inconsistent with lQCD.

We have found an appreciable sensitivity of the Euclidean correlator ratios to the so-called "reconstructed" (reference) spectral function used for normalization (usually taken as a vacuum form). The choice of shape and onset of the continuum introduces $\tau$-dependencies in the normalized correlator, which may render the identification of medium effects in the calculated spectral functions more difficult. E.g., when normalizing to a reference spectral function calculated at $1.5 T_{c}$ for the $S$-wave $c-\bar{c}$ system (as suggested by the corresponding 1QCD result), the lQCD correlators for higher temperatures can be much better reproduced. We have also inferred that the $1 Q C D$ correlators favor a temperature-dependent decrease of the heavy-quark mass $(Q-\bar{Q}$ threshold), which 
pushes spectral strength to lower energies and improves on the large- $\tau$ and temperature dependence of the correlators.

Our approach furthermore allows for establishing a closer connection to quarkonium phenomenology in heavy-ion collisions by incorporating finite width effects. E.g., when implementing in-medium heavy-quark widths of $\sim 50 \mathrm{MeV}$ in the two-particle propagator of the scattering equation (inducing a charmonium width of $\sim 100 \mathrm{MeV}$ as suggested by phenomenology), we find only few-percent changes in the Euclidean correlators, which are superseded by other current uncertainties.

In conclusion, our results suggest that 1QCD-based potential approaches, when consistently implementing both bound and scattering states in a non-perturbative scheme, are a viable means to quantitatively interpret the rather precise lQCD computations on Euclidean correlation functions, and thus evaluate the properties of quarkonium spectral functions in the QGP. Significant uncertainties still reside in the extraction of an appropriate $Q-\bar{Q}$ potential, as well as in the determination of the in-medium open-charm and -bottom masses. Once quantitative agreement between model calculations and 1QCD correlators has been established, applications to high-energy heavy-ion collisions will subject the theoretical results to experimental tests. Hopefully this will pave the way to progress on the long-standing challenge of connecting heavy-quarkonium observables to properties of the finite-temperature QCD phase transition.

\section{Acknowledgments}

We thank M. Mannarelli and F. Zantow for providing us with their (fits to) lattice QCD results. We also acknowledge useful discussions with M. Mannarelli and H. van Hees. One of us (DC) thanks Ministerio de Educación y Ciencia (Spain) for support through a postdoctoral fellowship. One of us (RR) has been supported in part by a U.S. National Science Foundation CAREER Award under Grant No. PHY0449489.

\section{APPENDIX A: 3-DIMENSIONAL REDUCTION OF THE BETHE-SALPETER EQUA-} TION

The Bethe-Salpeter equation for $Q-\bar{Q}$ scattering with the Blankenbecler-Sugar (BbS) threedimensional reduction of the two-particle propagator [15, 38] reads, in the CM frame,

$$
\mathcal{M}\left(E ; \vec{q}^{\prime}, \vec{q}\right)=\mathcal{V}\left(\vec{q}^{\prime}, \vec{q}\right)+\int \frac{d^{3} k}{(2 \pi)^{3}} \mathcal{V}\left(\vec{q}^{\prime}, \vec{k}\right) \frac{m^{2}}{\omega_{k}} \frac{\Lambda_{+}(\vec{k}) \Lambda_{-}(-\vec{k})}{s / 4-\omega_{k}^{2}+i \epsilon} \mathcal{M}(E ; \vec{k}, \vec{q}),
$$

where the invariant amplitudes $\mathcal{M}$ and $\mathcal{V}$ are actually operators (truncated amplitudes) which act in the direct product of the Dirac spaces of each fermion. The BbS scheme, originally formulated for the nucleon-nucleon $(N N)$ interaction, exploits the following decomposition of the single-particle propagator in terms of positive- and negative-energy states,

$$
S_{F}\left(k^{0}, \vec{k}\right)=\frac{m}{\omega_{k}} \frac{\Lambda_{+}(\vec{k})}{k^{0}-\omega_{k}+i \eta}-\frac{m}{\omega_{k}} \frac{\Lambda_{-}(-\vec{k})}{k^{0}+\omega_{k}-i \eta} .
$$

Consequently, the full four-dimensional two-particle propagator, $i S_{Q}(k+P / 2) S_{\bar{Q}}(k-P / 2)$, is replaced by the following function

$$
\delta\left(k^{0}\right) \frac{m^{2}}{\omega_{k}} \frac{\Lambda_{+}(\vec{k}) \Lambda_{-}(-\vec{k})}{s / 4-\omega_{k}^{2}+i \epsilon},
$$

which has the same discontinuity across the right-hand cut and puts the quark (anti-quark) on the positive (negative) energy shell, suppressing virtual anti-quark (quark) contributions. Note that 


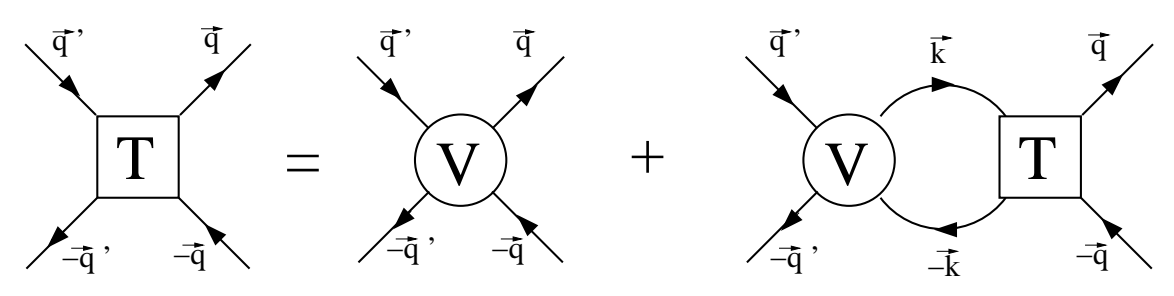

FIG. 17: CM kinematics of the Bethe-Salpeter equation.

since both fermions are equally off-shell the energy transfer at the interaction is zero (BbS neglects retardation effects), and this allows for a description in terms of a static potential, $V\left(\vec{q}^{\prime}, \vec{q}\right)$, as done for instance in the Boson Exchange Model of the $N N$ interaction [38].

One can take matrix elements in Eq. (A1) between the appropriate Dirac spinors, $\tilde{T}[\tilde{V}] \equiv$ $\bar{u}(\vec{q}) \bar{v}\left(-\vec{q}^{\prime}\right) \mathcal{M}[\mathcal{V}] u\left(\vec{q}^{\prime}\right) v(-\vec{q})$ (see Fig. 17 for kinematics), and then Eq. (A1) can be rewritten as (helicity indices omitted)

$$
\tilde{T}\left(E ; \vec{q}^{\prime}, \vec{q}\right)=\tilde{V}\left(\vec{q}^{\prime}, \vec{q}\right)+\int \frac{d^{3} k}{(2 \pi)^{3}} \tilde{V}\left(\vec{q}^{\prime}, \vec{k}\right) \frac{m^{2}}{\omega_{k}} \frac{1}{s / 4-\omega_{k}^{2}+i \epsilon} \tilde{T}(E ; \vec{k}, \vec{q}),
$$

where we have used the following representation of the energy projectors

$$
\begin{aligned}
\Lambda_{+}(\vec{k}) & =\frac{\sum_{\lambda} u_{\lambda}(\vec{k}) \bar{u}_{\lambda}(\vec{k})}{2 m}, \\
\Lambda_{-}(\vec{k}) & =\frac{-\sum_{\lambda} v_{\lambda}(-\vec{k}) \bar{v}_{\lambda}(-\vec{k})}{2 m} .
\end{aligned}
$$

The connection between $\tilde{T}, \tilde{V}$ and the actual (static) potential $V$ and the $T$-matrix in Eq. (1) can be derived by considering a tensor structure for $\tilde{V}$ and performing a non-relativistic reduction of the resulting amplitude (for instance consider $\tilde{V}$ given by the Yukawa scalar-meson exchange amplitude, which can be fully derived from the Lagrangian, $\left.\mathcal{L}_{S}=g_{S} \bar{\Psi} \phi\right)$. It turns out that $\tilde{V}\left(\vec{q}^{\prime}, \vec{q}\right)=V\left(\vec{q}^{\prime}, \vec{q}\right)+\mathcal{O}\left(q^{2} / m^{2}\right)$, with $V$ related to the corresponding potential in coordinate space by

$$
V(r)=\frac{1}{(2 \pi)^{3}} \int d^{3} k e^{i \vec{k} \vec{r}} V(\vec{k})
$$

and $\vec{k}=\vec{q}^{\prime}-\vec{q}$. The partial wave decomposition of the potential (and of $T$ ) is given by

$$
V\left(\vec{q}^{\prime}, \vec{q}\right)=4 \pi \sum_{l}(2 l+1) V_{l}\left(q^{\prime}, q\right) P_{l}\left(\cos \theta_{q^{\prime} q}\right)
$$

and then Eqs. (14) follow. The temperature dependence is accounted for by introducing a $\left[1-2 f^{Q}\right]$ factor for each two-particle loop, and the quark selfenergy enters the two-particle propagator by the replacement

$$
\left[s / 4-\omega_{k}^{2}+i \epsilon\right]^{-1} \longrightarrow\left[s / 4-\omega_{k}^{2}-2 i \omega_{k} \operatorname{Im} \Sigma\right]^{-1}
$$

in Eq. (A4), with $\omega_{k}$ satisfying the dispersion relation in Eq. (3). 
[1] N. Brambilla et al., arXiv:hep-ph/0412158.

[2] M. Asakawa and T. Hatsuda, Phys. Rev. Lett. 92, 012001 (2004).

[3] S. Datta, F. Karsch, P. Petreczky and I. Wetzorke, Phys. Rev. D 69, 094507 (2004)

[4] T. Umeda, K. Nomura and H. Matsufuru, Eur. Phys. J. C 39S1, 9 (2005).

[5] K. Petrov, A. Jakovac, P. Petreczky and A. Velytsky, PoS LAT2005, 153 (2006).

[6] E. V. Shuryak and I. Zahed, Phys. Rev. C 70, 021901 (2004).

[7] C. Y. Wong, Phys. Rev. C 72, 034906 (2005).

[8] W. M. Alberico, A. Beraudo, A. De Pace and A. Molinari, Phys. Rev. D 72, 114011 (2005).

[9] A. Mocsy and P. Petreczky, Phys. Rev. D 73, 074007 (2006).

[10] M. Mannarelli and R. Rapp, Phys. Rev. C 72, 064905 (2005).

[11] L. Grandchamp, R. Rapp and G.E. Brown, Phys. Rev. Lett. 92, 212301 (2004).

[12] L. Grandchamp, S. Lumpkins, D. Sun, H. van Hees and R. Rapp, Phys. Rev. C 73, 064906 (2006).

[13] R. Rapp, Eur. Phys. J. A 18, 459 (2003).

[14] A. Mocsy and P. Petreczky, Eur. Phys. J. C 43, 77 (2005).

[15] R. Blankenbecler and R. Sugar, Phys. Rev. 142, 1051 (1966).

[16] M. I. Haftel and F. Tabakin, Nucl. Phys. A158, 1 (1970).

[17] Z. Aouissat, R. Rapp, G. Chanfray, P. Schuck and J. Wambach, Nucl. Phys. A 581, 471 (1995).

[18] M. Schmidt, G. Röpke and H. Schulz, Ann. Phys. 202, 57-99 (1990).

[19] M. Mannarelli and R. Rapp, in preparation (2006).

[20] H. van Hees and R. Rapp Phys. Rev. C 71, 034907 (2005).

[21] H. van Hees, V. Greco and R. Rapp, Phys. Rev. C 73, 034913 (2006).

[22] S. Digal, P. Petreczky, and H. Satz, Phys. Rev. D 64, 094015 (2001).

[23] O. Kaczmarek, F. Karsch, P. Petreczky, and F. Zantow, Nucl. Phys. Proc. Suppl. 129, 560 (2004).

[24] P. Petreczky, private communcation (2004).

[25] O. Kaczmarek and F. Zantow, arXiv:hep-lat/0506019.

[26] C. Y. Wong and H. W. Crater, arXiv:hep-ph/0610440.

[27] H. Satz, arXiv:hep-ph/0602245.

[28] G. E. Brown, Philos. Mag. 43, 467 (1952).

[29] C. Y. Wong, arXiv:hep-ph/0606200.

[30] F. Karsch, M.G. Mustafa and M.H. Thoma, Phys. Lett. B 497, 249 (2001).

[31] W. M. Alberico, A. Beraudo, and A. Molinari, Nucl. Phys. A750, 359 (2005).

[32] G. T. Bodwin, E. Braaten and G. P. Lepage, Phys. Rev. D 51, 1125 (1995) [Erratum-ibid. D 55, 5853 (1997)].

[33] A. Gara, B. Durand, L. Durand and L. J. Nickisch, Phys. Rev. D 40, 843 (1989).

[34] E. V. Shuryak, Rev. Mod. Phys. 65, 1 (1993).

[35] R. Rapp, D. Cabrera and H. van Hees, arXiv:nucl-th/0608033.

[36] D. Cabrera and R. Rapp, arXiv:hep-ph/0610254

[37] H. Iida, T. Doi, N. Ishii, H. Suganuma and K. Tsumura, arXiv:hep-lat/0602008

[38] R. Machleidt, Adv. Nucl. Phys. 19, 189 (1989). 